Pacific Journal of Mathematics

DEFORMATION OF UNIFORM ALGEBRAS ON RIEMANN 


\title{
DEFORMATION OF UNIFORM ALGEBRAS ON RIEMANN SURFACES
}

\author{
RICHARD ROCHBERG
}

\begin{abstract}
The main theme of this paper is the study of algebras of analytic functions of finite bordered, possible singular, Riemann surfaces from the point of view of the deformation theory of uniform algebras as introduced in earlier papers by the author.
\end{abstract}

I. Introduction. We begin by recalling some results from [15]. Let $A$ be a uniform algebra with maximal ideal space $M(A)$ and Shilov boundary $\partial A$. We assume further that

every point of $\partial A$ is a strong peak point

(i.e., if $p$ is in $\partial A$ then there is an $f$ in $A$ with $\|f\|=f(p)$ and $|f(q)|<1$ for all $q, q \neq p$.) (The role of this restriction is discussed in §2.) A deformation of $A$ is a new normed algebra obtained by putting on the vector space $A$ a new commutative associative multiplication, $\times$, which, for some small positive $\varepsilon$, satisfies

$$
\|f \times g-f g\| \leq \varepsilon\|f\|\|g\| \text { for all } f, g \text { in } A .
$$

If $\varepsilon$ is small and this new algebra is renormed with its spectral norm then we obtain a new uniform algebra, $A_{\times}$, which also satisfies (1.1). If $A$ is separable then $A_{\times}$will also be separable. We summarize this construction by saying that $A_{\times}$is an $\varepsilon$-deformation of $A$.

We regard two uniform algebras as equivalent if each is isomorphic to an arbitrarily small deformation of the other. Let $\mathfrak{M}$ be the space of separable uniform algebras which satisfy (1.1) modulo the equivalence relation just described. (This equivalence relation does not actually identify distinct algebras in the class we will be considering (Proposition 2.9).)

The Banach-Mazur distance between Banach spaces is an (extended valued) metric on $\mathscr{M}$. It is shown in [15] that for nearby points $A_{1}, A_{2}$ in $\mathscr{M}$ the distance between $A_{1}$ and $A_{2}$ is comparable to the smallest $\varepsilon$ such that $A_{1}$ is isomorphic to an $\varepsilon$ deformation of $A_{2}$. It is also shown that $\mathscr{M}$ is a complete metric space.

Let $\mathscr{S}$ be the set of all connected finite bordered Riemann surfaces. For $S$ in $\mathscr{S}$, let $A(S)$ be the supremum normed Banach algebra of functions continuous on $S$ and analytic at the non-boundary points of $S$. 
All such $A(S)$ are in $\mathscr{M}$. Hence the topology on $\mathscr{M}$ induces a topology on $\mathscr{S}$. Using the results of [15] and [12], it is known that this topology is the classical moduli topology on $\mathscr{S}$ (i.e., the topology induced by the Teichmüller metric). It is also known that the set $A(\mathscr{S})=\{A(S) ; S \in \mathscr{S}\}$ is not a closed subset of $\mathscr{M}$. For example, let $D$ be the closed unit disk and let $A_{0}=\left\{f \in A(D), f^{\prime}(0)=0\right\}$. The constructions in [14] show that $A_{0}$, regarded as an element of $\mathscr{M}$, is in the closure of $A(\mathscr{S})$.

With this and similar examples as motivation, we introduce a larger class of algebras in $\mathscr{M}$. Let $\mathscr{A}$ be the collection of algebras $A$ in $\mathscr{M}$ for which $M(A)$ is connected and which can be obtained as subalgebras of finite codimension of finite direct sums of algebras in $A(\mathscr{S})$. In addition we require that $\partial A$ be (homeomorphic to) a finite union of circles. (The purpose of this restriction is discussed in \$2.) Hence, in particular, $A(\mathscr{S}) \subseteq \mathscr{A}$. Informally, these algebras are algebras of the form $A(S)$ where $S$ is a finite, bordered, connected, singular Riemann surface. These algebras are described fully in $\S 2$. Associated with any $a$ in $\mathscr{A}$ is an integer, the defect of $A$, which is defined to be the dimension of the space of real measures on $\partial A$ which annihilate $A$. Let $\mathscr{A}_{k}=\{A \in \mathscr{A}$; the defect of $A$ is $k\}$. For example, if $S$ is in $\mathscr{S}$, the genus of $S$ is $g$ and the boundary of $S$ has $c$ components then $A(S)$ is in $\mathscr{A}_{k}$ for $k=2 g+c-1$. Another example is the algebra $A_{0}$ just described; $A_{0}$ is in $\mathscr{A}_{2}$.

Our main results concern the set $\mathscr{A}$ regarded as a subset of $\mathscr{M}$.

Section 3 contains results which show that if $A$ is in $\mathscr{A}$ and $B$ is a small deformation of $A$ then $B$ has many of the properties of elements of $\mathscr{A}$. When these results are combined with Theorem 6.3 of [15] (which shows that "defect of $A$ " is a cotinuous function on $\mathscr{M}$ ) we obtain our two main results about the global geometry of $\mathscr{A}$. Corollary 3.6 states that each $\mathscr{A}_{k}$ is an open subset of $\mathscr{M}$. (This generalized the main result of [16].) Corollary 3.7 states that each $\mathscr{A}_{k}$ is a closed subset of $\mathscr{M}$ (and hence is also a complete metric space).

In $\S 4$ we study the local structure of $\mathscr{A}_{k}$. That is, we study which algebras can occur in small neighborhoods of a given $A$ in $\mathscr{A}_{k}$. Corollary 7.7 of [15] states that the function "dimension of the first cohomology group (over R) of $M(A)$ " is lower semi-continuous on $\mathscr{A}_{k}$. Since this dimension is at most $k$ on $\mathscr{A}_{k}$ and equals $k$ exactly on $\mathscr{A}_{k} \cap A(\mathscr{S})$ we conclude that $\mathscr{A}_{k} \cap A(\mathscr{S})$ is an open subset of $\mathscr{A}_{k}$ (and hence $A(\mathscr{S})$ is an open subset of $\mathscr{M}$ ). Combining this with the results of [15] and [12] we conclude that small neighborhoods of algebras $A(S), S$ in $\mathscr{S}$, consist exactly of algebras $A\left(S^{\prime}\right)$ with $S^{\prime}$ in $\mathscr{S}$ and $S^{\prime}$ quasiconformally equivalent 
to $S$ by a quasiconformal map with dilatation close to one. For algebras $A$ in $\mathscr{A}_{k} \backslash\left(\mathscr{A}_{k} \cap A(\mathscr{S})\right)$, i.e., algebras of functions on singular Riemann surfaces, the situation is more complicated. The structure of such algebras is determined by the conformal structure of $M(A)$ and by the behavior of the functions in $A$ in neighborhoods of a certain finite set, which we denote $\operatorname{Sing}(A)$, in $M(A)$. ( $\operatorname{Sing}(A)$ is the set of singular points of the singular surface on which functions in $A$ are defined.) The main technical result of $\S 4$ is Theorem 4.2 which shows that if we start with such an $A$ and any small neighborhood $N$ of $\operatorname{Sing}(A)$ then for any $B$ which is sufficiently close to $A$, there is a quasiconformal map with dilatation close to one which maps $M(A) \backslash N$ into $M(B)$ (and has certain other nice properties). Informally, the small deformation of $A$ produces two types of changes. The first is the global change in the conformal structure of $M(A)$ away from $\operatorname{Sing}(A)$. This is described by Theorem 4.2. The second type of change is the localized deformation of the structure of $M(A)$ near the singular points. These changes are described in detail in $\$ 4$ for the simplest types of singularities (nodes and cusps). These results are then combined to give fairly detailed descriptions of $\mathscr{A}_{1}$ and $\mathscr{A}_{2}$.

The survey by Palamodov [9] and the references there indicate the large variety of approaches which have been taken to problems of deformation of and moduli for complex structures. Our results are in the same spirit as many of those described in [9]. However, there is a substantial difference between our point of view and that described in [9] which prevents a complete analogy of results and prevents our adapting many of the techniques described there. The difference is that our notion of small deformation does not carry with it a clearly associated notion of infinitesimal deformation (i.e., our moduli space $\mathscr{M}$ is a metric space which lacks $a$ priori differentiable structure). In contrast, most of the results in [9] are formulated in terms of a family of deformations of a fixed object and often involve infinitesimal analysis of the family at the base point. For example, the informal idea that a small deformation of $A$ in $\mathscr{A}$ consists of a global deformation of conformal structure and a local deformation at isolated singularities is not formulated here as a direct sum decomposition of an associated space of germs of deformations. The results in [4], [6] and [10] suggest that it may be possible to develop a theory of infinitesimal deformations of elements of $\mathscr{M}$; however this remains to be investigated.

We will make free use of the theory of uniform algebras as found in [2], [17] and [18]. In particular we will identify elements of a uniform algebra with Gelfand transforms. 
We will denote the space of invertible linear maps between the Banach spaces $A$ and $B$ by $L(A, B)$.

Some of these results were announced in [13].

A good introduction to these topics is given by Jarosz in [19].

II. Algebras in $\mathscr{A}$. For any $S$ in $\mathscr{S}$, let $\partial S$ be the border of $S$.

We now describe three ways to obtain elements of $\mathscr{A}$ from elements of $A(\mathscr{S})$. First, let $S$ be in $\mathscr{S}$ and let $p$ and $q$ be distinct points of $S \backslash \partial S$. The subalgebra of codimension one of $A(S)$ consisting of functions $f$ in $A(S)$ for which $f(p)=f(q)$ is an element of $\mathscr{A}$. Second, let $p$ be a point of $S \backslash \partial S$. Let $D$ be a point derivation at $p$; that is, $D$ is a continuous linear functional which satisfies

$$
D(f g)=f(p) D(g)+g(p) D(f)
$$

for all $f, g$ in $A$. (Equivalently $D(1)=0$ and $D$ vanishes on the square of the ideal of functions which vanish at $p$.) If $D$ is not identically zero, then the kernel of $D$ is a subalgebra of $A(S)$ of codimension one and is an element of $\mathscr{A}$. Finally, let $S_{l}$ be in $\mathscr{S}$ for $i=1,2$ and select $p_{\imath}$ in $S_{\imath} \backslash \partial S_{l}$. The subalgebra of $A\left(S_{1}\right) \oplus A\left(S_{2}\right)$ consisting of functions $f$ which satisfy $f\left(p_{1}\right)=f\left(p_{2}\right)$ is a subalgebra of codimension one of $A\left(S_{1}\right) \oplus A\left(S_{2}\right)$ and is an element of $\mathscr{A}$.

Gamelin has given a complete description of subalgebras of finite codimension of uniform algebras [3]. His results applied in this context show that repeated application of the three constructions just described gives all elements of $\mathscr{A}$. Here is a summary of his results for the case of interest to us.

Proposition 2.1. Suppose $A$ is in $\mathscr{A}_{k}$. There are $S_{l}$ in $\mathscr{S}, i=1, \ldots, N$, a subalgebra $B$ of $A\left(S_{1}\right) \oplus A\left(S_{2}\right) \oplus \cdots \oplus A\left(S_{N}\right)$, and subalgebras $A_{i}$ of $B$, $i=1, \ldots, M$, so that

(a) $A=A_{1} \subseteq \cdots \subseteq A_{M}=B$

(b) $B$ is obtained from $A\left(S_{1}\right) \oplus A\left(S_{2}\right) \oplus \cdots \oplus A\left(_{N}\right)$ by making finitely many point identifications of points selected from the $S_{\iota} \backslash \partial S_{i} . M(B)$ is the singular connected bordered Riemann surface obtained from $S_{1} \cup$ $S_{2} \cup \cdots \cup S_{N}$ by making those point identifications. $\partial B=\cup \partial S_{i}$

(c) $M(A)=M\left(A_{i}\right)=M(B) ; \partial A=\partial A_{l}=\partial B, i=1, \ldots, M$.

(d) Each $A_{i}$ is obtained from $A_{i+1}$ by taking the kernel of a point derivation at some point $q_{i}$ in $M\left(A_{i+1}\right) \backslash \partial A_{t+1}=M(B) \backslash \partial B$.

(e) There is an integer $J$ which depends only on $k$ such that if $f$ is in $B$ and $f\left(q_{l}\right)=0$ for $i=1, \ldots, M-1$, then the ideal $f^{J} B$ is contained in $A$. 
Proof. The only statement that is not immediate from the results in [3] is that $J$ can be chosen to depend only on $k$. However, it is shown in [3] that $J$ can be chosen to depend only on $M$ and simple topological considerations show that $k$ dominates $M$. (See Proposition 2.5 below.)

In particular, note that the conditions describing $A$ are local. That is,

Corollary 2.2. Suppose $f$ is in $A\left(S_{1}\right) \oplus \cdots \oplus A\left(S_{N}\right)$ and that each $p$ in $\bigcup S_{\imath} \backslash \cup \partial S_{i}$ has a neighborhood on which $f$ is a uniform limit of elements of $A$, then $f$ is in $A$.

The construction described in part (d) of the proposition is easy to understand if the maximal ideal $M_{t+1}$ of functions in $A_{\imath+1}$ which vanish at $q_{l}$ is principal. In that case, the dimension of $M_{i+1} / M_{i+1}^{2}$ is one and the space of derivations at $q_{i}$ is one dimensional. $D(f)$ will equal $c f^{\prime}\left(q_{i}\right)$ for some constant $c$ (which depends on the choice of local uniformizer). If the ideal is not principal, the situation can be more complicated. Here is the first non-trivial example of that. Let $A_{0}$ be the subalgebra of the disk algebra introduced in $\S 1$. For $f$ in $A_{0}$ let $D_{2} f=f^{\prime \prime}(0)$ and $D_{3} f=f^{\prime \prime \prime}(0)$. These are linearly independent point derivations of the algebra $A_{0}$ at the point 0 (and together they span the full space of such). The kernels of $D_{2}$ and $D_{3}$ are non-isomorphic subalgebras of $A_{0}$. Other subalgebras of $A_{0}$ are obtained by taking the kernel of $\alpha D_{2}+\beta D_{3}$ for other choices of $\alpha, \beta$. Even if we restrict attention to subalgebras of the disk algebra obtained by taking kernels of a sequence of point derivations at the origin, the situation rapidly becomes quite complicated and involves the algebraic theory of curve singularities.

For $A$ in $\mathscr{A}_{k}$ we will often speak of $M(A)$ as a Riemann surface. We will sometimes emphasize this point of view by writing $S(A)$ for $M(A)$, and $\partial S(A)$ for $\partial A$. Thus if $S$ is in $\mathscr{S}$ then $S(A(S))=S$.

There are four variations on this construction which are not described in Proposition 2.1. First, we did not allow $q_{i}$ to be a boundary point. The reason for this is simply that there are no non-zero point derivations at points on $\partial B$. This follows, for instance, from the fact that each such point is a strong peak point (see, e.g., page 88 of [5]). Second, we insisted that $M(B)$ be connected. This forces a non-trivial lower bound on the number of point identifications which are made on the disconnected surface $U S_{i}$. This is only a technical convenience. The entire theory of this paper extends with no surprises to finite direct sums of algebras in $\mathscr{A}$. (The proof of Theorem 5.3 of [16] shows how to reduce to analysis of individual summands.) The other two possibilities which were not presented were 
identification of points of $\bigcup \partial S_{i}$ with points of $U S_{i} \backslash \bigcup \partial S_{i}$ or with other points of $\bigcup \partial S_{i}$. Such identifications would produce subalgebras of finite codimension of direct sums of algebras in $A(\mathscr{S})$. In the first case, the resulting algebra would violate (1.1). (The identified point would not be a strong peak point.) In the second case, $\partial A$ would not be a disjoint union of circles. Thus, in neither case would the resulting algebras be in $\mathscr{A}$. The reason for defining $\mathscr{A}$ so as to exclude such algebras is that although the algebras involved are interesting from the point of view of deformation theory, the techniques for studying them would have to be slightly different from the ones we will be using.

Informally, the surfaces $S(A), A$ in $\mathscr{A}$, can be thought of as connected bordered subsets of singular Riemann surfaces. The requirement that the points of $\cup \partial S_{i}$ not be involved in the point identifications is the requirement that the singularities of the surface not be at boundary points. The singular surface may have compact components. These components are invisible in $M(A)$ but have shadows, namely the restrictions on the functions in $A$ at the $q_{i}$. If a small deformation of $A$ should desingularize the point $q_{i}$ then the component may become visible. (For further discussion from this point of view, see [14] and $\$ 12$ of [9].)

We refer to the set of all the $q_{i}$ together with all the identified points of the various $S_{i}$ as the singular set of $A$, denoted $\operatorname{Sing}(A)$. The two simplest types of points of $\operatorname{Sing}(a)$ are those which were obtained by an identification of exactly two points of the $\cup S_{i}$ which are not among the $q_{i}$, and those which are not among the identified points and which appear exactly once on the list of the $q_{i}$. We will refer to such points as nodes and cusps, respectively. Analysis of the ideal of functions in $A$ which vanish at such points (this is done in §4) shows that this terminology is parallel to that used in describing isolated singularities of algebraic curves.

The points of $M(A) \backslash(\partial A \cup \operatorname{Sing}(A))$ are points where the functions in $A$ have simple behavior.

Proposition 2.3. Suppose $A$ is in $\mathscr{A}$ and $p$ is in

$$
M(A) \backslash(\partial A \cup \operatorname{Sing}(A)) .
$$

The maximal ideal of functions in $A$ which vanish at $p$ is principal.

Proof. First we must find an $f$ in $A$ which vanishes only at $p$. Using the properties of point derivations ([3]), we see that this will be accomplished if we find an $f$ in $A\left(S_{1}\right) \oplus \cdots \oplus A\left(S_{n}\right)$ which takes the value 1 at all of the points which will be identified, vanished at $p$ and no other point, and has derivative which vanishes to a sufficiently high order (in terms of 
every applicable local coordinate system) at the points associated with the point derivations. First find $f_{0}$ which takes the right values then let $f=f_{0} e^{k}$ where $k$ is selected in $B$ to give the approximate vanishing of the derivative. To see that such an $f$ actually generates the ideal, we need the following lemma which we will also use later.

Lemma 2.4. We use the notation of Proposition 2.1. Suppose $h$ is in $A, g$ is in $B$ and $h g$ is in $A$. Suppose further that $h(q) \neq 0$ for all $q$ in $\operatorname{Sing}(A)$. Then $g$ is in $A$.

Proof of Lemma. We show that, in the notation of Proposition 2.1, if $g$ is in $A_{i+1}$ then $g$ is in $A_{i}$. Let $D$ be the point derivation at $q_{i}$ whose kernel is $A_{i}$

$$
D(h g)=h\left(q_{i}\right) D(g)+g\left(q_{i}\right) D(h) .
$$

Since $h g$ is in $A, D(h g)=0$. Since $h$ is in $A, D(h)=0$. Hence $h\left(q_{i}\right) D(g)$ $=0$. Since $q_{i}$ is in $\operatorname{Sing}(A), h\left(q_{i}\right) \neq 0$. Thus $D(g)=0$ and hence $g$ is in $A_{i}$.

To finish the proof of the proposition take $F$ in $A$.

$$
F=F(p)+\frac{F-F(p)}{f} f .
$$

By the lemma, $(F-F(p)) / f$ is in $A$. Hence $f$ generates the ideal.

We note that the converse is also true. The points of $M(A)$ for which the maximal ideals are not principal are exactly $\operatorname{Sing}(A) \cup \partial A$.

Associated with $A$ in $\mathscr{A}$ are two integers. The first $k=k(A)$, the dimnsion of $(\operatorname{Re} A)^{\perp}$, the space of real measures on $\partial A$ which annihilate $A$. $A$ is in $\mathscr{A}_{k}$ for $k=k(A)$. The second integer is the dimension of the real cohomology group $H^{1}(M(A), \mathbf{R})$. (The discussion of the significance of this group in our context is in [15].) These two integers are denoted $\operatorname{dim} X(A)$ and $\operatorname{dim} Y(A)$ in [15]. Suppose $A$ is as described in Proposition 2.2. For $i=1, \ldots, N$ let $g_{i}$ be the genus of $S_{i}$ and $c_{i}$ the number of components of $\partial S_{l}$. Let $g=\sum_{i=1}^{N} g_{i}$ and let $c=\sum_{i=1}^{N} c_{i}$. Hence $c$ is the number of components of $\partial A$. Let $R$ be the number of point identifications made in passing to the algebra $B$. Since we required $M(A)$ to be connected, we must have $R \geq N-1$. Let $D(=M-1)$ be the number of kernels of point derivations which must be taken to pass from $B$ to $A$.

\section{Proposition 2.5.}

$$
k(A)=2 g+c+2 R+2 D+1-2 N
$$

$$
\operatorname{dim} H^{1}(M(A), \mathbf{R})=2 g+c+R+1-2 N \text {. }
$$


In particular $k(A)-c$ is odd. Also

$$
k(A)=\operatorname{dim} H^{1}(M(A), \mathbf{R})
$$

holds if and only if $R=D=0, N=1$. Thus (2.3) holds if and only if $A=A(S)$ for some $S$ in $\mathscr{S}$.

Proof. First note that for each $i, k\left(A\left(S_{i}\right)\right)=2 g_{i}+c_{i}-1$. Hence $\operatorname{dim} \operatorname{Re}\left(A\left(S_{i}\right) \oplus \cdots \oplus A\left(S_{N}\right)\right)^{\perp}=2 g+c-N$. Next note that if $A_{1}$ and $A_{2}$ are in $M$ and $A_{3}$ is the subalgebra of codimension one of $A_{1} \oplus A_{2}$ obtained by identifying a point of $M\left(A_{1}\right)$ with a point of $M\left(A_{2}\right)$ then $\operatorname{dim}\left(\operatorname{Re} A_{3}\right)^{\perp}=\left(\operatorname{dim} \operatorname{Re}\left(A_{1} \oplus A_{2}\right)^{\perp}\right)+1$. Hence if we start with the $S_{1}, \ldots, S_{N}$ and make $N-1$ of the $R$ point identifications to obtain a connected set and denote the resulting subalgebra of $A\left(S_{1}\right) \oplus \cdots \oplus A\left(S_{N}\right)$ by $B_{1}$, then $\operatorname{dim}\left(\operatorname{Re} B_{1}\right)^{\perp}=2 g+c-1$. The algebra $A$ is obtained from the algebra $B_{1}$ in $(R-(N-1))+D$ steps each of which involves passage to a subalgebra of codimension one of the algebra obtained at the previous step. We will show that each such step increases $k(\cdot)$ by two. We will describe the analysis at the first step. (The general step is the same.) The passage from $B_{1}$ to the next algebra $B_{2}$ involves taking the kernel of a linear functional $r$. ( $r$ is either a point derivation of a difference of point evaluations.) Furthermore, the functional $r$ annihilates constants. Since $M\left(B_{1}\right)$ is connected, a function $f$ in $B_{1}$ is determined up to an additive constant by the values of $\operatorname{Re} f$ on $\partial B_{1}$. Hence the value of $r(f)$ is a linear functional on the set of functions on $\partial B_{1},\left\{\operatorname{Re} f, f \in B_{1}\right\}$. Since $r$ only involves the values of $f$ at interior points this functional in continuous. Hence there is a complex measure $\mu$ on $\partial B_{1}$ such that

$$
r(f)=\int_{\partial B_{1}}(\operatorname{Re} f) d \mu .
$$

Let $\mu_{1}$ and $\mu_{2}$ be the real and imaginary parts of $\mu$. It is straightforward to check that $\mu_{1}$ and $\mu_{2}$ are linearly independent, are in $\operatorname{Re} B_{2}^{\perp}$, and that, in fact, $\mu_{1}$ and $\mu_{2}$ are a basis of $\left(\operatorname{Re} B_{2}^{\perp}\right) /\left(\operatorname{Re} B_{1}^{\perp}\right)$. Combining these observations establishes (2.1).

(2.2) is an elementary exercise in algebraic topology.

The surfaces $S(A)$ for $A$ in $\mathscr{A}$ do not generally have globally defined coordinate functions. The following proposition gives partial substitutes for coordinate functions. Ahlfors [1] has shown that given $S$ in $\mathscr{S}$ and $x, y$ in $S \backslash \partial S$ there is a function $F=F_{x, y}$ in $A(S)$ which has $|F|=1$ identically on $\partial S, F(x)=0, F(y) \neq 0$, and $F$ maps $S$ onto the closed unit disk 
in an $m$ to one manner (counting multiplicity). Furthermore, if $g$ denotes the genus of $S$ and $c$ the number of components of $\partial S$, then $F$ can be selected so that $m$ satisfies

$$
c \leq m \leq 2 g+c .
$$

Proposition 2.5. Suppose $A$ is in $\mathscr{A}_{k}, S=S(A), x$ and $y$ are distinct points of $S \backslash \partial S$, and $y$ is not in $\operatorname{Sing}(A)$. Let $S_{1}, \ldots, S_{n}$ be the components of $S$ (prior to the point identifications) and let $\tilde{A}=A\left(S_{1}\right) \oplus \cdots \oplus A\left(S_{n}\right)$. There is $a \mathrm{G}$ in $A$ so that

(a) $G(x)=0, G(y) \neq 0,|G(t)|=1$ for all $t$ in $\partial S$.

(b) Let $C+G \tilde{A}=\{f \in \tilde{A} ; f=c+G g, c \in C, g \in \tilde{A}\} . \tilde{A} \supseteq A \supseteq C+$ $G \tilde{A}$.

(c) There are a positive $\varepsilon$ and an integer $M$ (both may depend on $x, y$ ) so that $G$ is an $M$ to 1 unbranched map of a neighborhood of $\partial S$ onto $\{z$; $1-\varepsilon<|z| \leq 1\}$.

(d) There is an integer $K$ which depends only on $k$ such that $M \leq K$.

Proof. Let Sing $A=\left\{w_{1}, \ldots, w_{r}\right\}$. Let $G_{1}=F_{x, y} \partial F_{w_{i}, y}$. Let $J$ be the integer in (3) of Proposition 2.1. Let $G=G_{1}^{J}$. Parts (a), (b), and (c) follow from the properties of the $F$ 's and from (e) of Proposition 2.2. The estimate on $M$ follows from the fact that $r$ can be estimated in terms of $k$ (by (2.1)), the valance of each $F$ can be estimated in terms of $k$ (by (2.4) and (2.1) (note that the letters $g$ and $c$ are used differently in those two equations)) and the estimate on $J$ is (e) of Proposition 2.1.

We will want to be able to separate points near $\partial A$ which are identified by such a $G$.

Proposition 2.6. Let $A$ in $\mathscr{A}_{k}, p$ in $\partial A$ and $\eta>0$ be given. There are neighborhoods $N_{1}, N_{2}$ of $p ; p \in N_{1} \subseteq N_{2}$ and a function $F$ in $A,\|F\|=1$ so that

(a) $F$ is a one-to-one map of $N_{1}$ to $D_{+}=D \cap\{\operatorname{Re} z>0\} ; F^{-1}\left(D_{+}\right)=N_{1}$.

(b) ||$F(t)|-1|<\eta$ for all $t$ in $\partial A$.

(c) $|F+1|<\eta$ on $M(A) \backslash N_{2}$.

Proof. Start with a function $G$ of the sort described in the previous proposition. Select $G$ so that $G(p)=1$. Let $J=\left\{e^{i \theta} ;|\theta|<\pi / 10\right\}$. Let $I$ be the component of $G^{-1}(J)$ which contains $p$. Let $H$ be a function which is analytic on $M(A)$, of modulus one on $\bar{I}$, of modulus strictly less than one on $\partial A \backslash \bar{I}$ and has $H(p)=1$. (Such an $H$ can be obtained, for instance, by the construction in the previous proposition applied to a surface $S^{+}$which contains $M(A)$.) By (b) of the previous proposition, 
$G H=G_{1}$ is in $A$. Let $I_{1}$ be the component of $G_{1}^{-1}(J)$ which contains $p$. Let $J^{\prime}=G_{1}\left(I_{1}\right)$. Let $R$ be a function in the disk algebra which has $|R|=1$ on $J^{\prime} ;|R|<1$ on $D \backslash J^{\prime}$, the variation of $\arg R$ on $R^{\prime}$ is less than $\pi / 4$, and $R(1)=1$. ( $R$ could be obtained, for instance, as a conformal map.) Since the polynomials are dense in the disk algebra, the composite function $G_{2}=R \circ G_{1}$ is in $A$. By construction $\left|G_{2}\right|=1$ on $\bar{I}_{1},\left|G_{2}\right|<1$ on $M(A) \backslash \bar{I}_{1}$, and $G_{2}$ is a one-to-one map of a neighborhood of $p$ onto a neighborhood of 1 in $D$. For a small positive $\delta$, let $G_{\delta}$ be the composition of $G_{2}$ with the conformal map of $D$ to itself which fixes 1 and sends $1-\delta$ to 0

$$
G_{\delta}=(G-(1-\delta))(1-(1-\delta) G)^{-1} \text {. }
$$

It is now straightforward that for sufficiently small $\delta, G_{\delta}$ is the required function $F$. We set $N_{1}=G_{\delta}^{-1}\left(D_{+}\right)$and $N_{2}=G_{\delta}^{-1}(\{z ;|1+z|<\eta\})$.

In order to apply certain results of [15] we need the following.

Proposition 2.7. Suppose $A$ is in $\mathscr{A}$. There is an $\varepsilon=\varepsilon(A)$ so that if $f$ in $A$ is invertible (i.e., $f \in A^{-1}$ ) and $\|\log |f|\|_{\infty}<\varepsilon$ then $f=e^{g}$ for some $g$ in $A$ (i.e., $f$ is in $e^{A}$ ).

Proof. We use the notation of Proposition 2.1. The algebra $A\left(S_{1}\right) \oplus$ $\cdots \oplus A\left(S_{N}\right)$ satisfies the conclusion of the Proposition (since each summand does.) Thus, if $\|\log |f|\|_{\infty}<\varepsilon$, then there is a $g$ in $A\left(S_{1}\right) \oplus \cdots \oplus$ $A\left(S_{N}\right)$ so that $f=e^{g}$. We next show that if $\varepsilon$ is smaller still, we can insure that $g$ is in $B$. Let $p, q$ be two points of $\cup S_{i}$ which are not separated by $B$. Since $e^{g}$ is in $B$ we have

$$
g(p)-g(q)=2 \pi \text { in }
$$

for some integer $n$. However, $\operatorname{Re} g=\log |f|$. Let $S=S(A)$. We use the following result.

Lemma. Given $p$, $q$ in $S \backslash \partial S$ there is an $\varepsilon^{\prime}>0, \varepsilon^{\prime}=\varepsilon^{\prime}(p, q, S)$ such that if $H$ is continuous on $S$ and analytic on $S \backslash \partial S$ and $\|\operatorname{Re} H\|_{\infty}<\varepsilon^{\prime}$ then $|H(p)-H(q)|<1$.

\section{Proof. Omitted.}

By using this lemma, we can insure that, if $\varepsilon$ is small, then $n=0$ in (2.5). Doing this for each such pair $p, q$ will insure that $g$ is in $B$. In fact, $g$ is in $A$. Although this can be shown by elementary means, we invoke the general Banach algebra result (Corollary 8.22 in [17]) which insures that since $f$ has a continuous $\log$ arithm on $M(A)$ (i.e., $\log f=g$ ) then $f$ must be the exponential of some $h$ in $A$ (and hence $h=g$ ). 
If $A$ is in $\mathscr{A}_{k}$ then, by definition, $\operatorname{dim} \operatorname{Re} A^{\perp}=k . A$ is clearly antisymmetric. Combining these facts with the previous proposition, we have, using the notation of [15]

\section{Corollary 2.8. $\mathscr{A}_{k} \subseteq N_{k}$.}

Thus the continuity results of $\S \S 6$ and 7 of [15] apply to the $\mathscr{A}_{k}$.

Finally we show that distinct elements of $\mathscr{A}$ yield distinct elements of $\mathscr{M}$. We don't use this result later and the proof could be postponed to $\S 4$. We include it now to emphasize that it is independent of the later results and because the proof uses different types of considerations from those used in the later sections.

Proposition 2.9. Suppose $A_{1}, A_{2}$ are in $\mathscr{A}$ and each is isomorphic to an arbi: $r$ rarily small deformation of the other, then $A_{1}=A_{2}$.

Proof. In the case when $A_{i}=A\left(S_{i}\right), S_{i} \in \mathscr{S}$ for $i=1,2$ it suffices to show that $S_{1}$ and $S_{2}$ are conformally equivalent. This is Proposition 5 of [11]. That proof can be used in this context unless both $M\left(A_{1}\right)$ and $M\left(A_{2}\right)$ are the disk (we consider that case later). Straightforward extension of the proof of Proposition 5 of [11] shows that $M\left(A_{1}\right)$ and $M\left(A_{2}\right)$ are conformally equivalent (possibly singular) Riemann surfaces. Denote both by $S$ and let $A(S)$ be the algebra of continuous functions on $S$ which are holomorphic on $S \backslash \partial S$. We can regard both $A_{1}$ and $A_{2}$ as subalgebras of $A(S)$. We also obtain from that proof that there is a sequence of linear maps $T_{n}$ of $A_{1}$ to $A_{2}$ and a conformal automorphism $\tau$ of $S \backslash \partial S$ so that for all $f$ in $A_{1}, T_{n} f$ converges to $f \circ \tau$, uniformly on compact subsets of $S \backslash \partial S$. Since $\tau$ extends continuously to $S, f \circ \tau$ is in $A(S)$. Since $f \circ \tau$ is in $A(S)$ and is the uniform limit on compact subsets of elements of $A_{2}$. We may use Corollary 2.2 and conclude that $f \circ \tau$ is in $A_{2}$. Thus the map of $f$ to $f \circ \tau$ is an algebra automorphism of $A(S)$ which takes $A_{1}$ into $A_{2}$. Hence $\operatorname{dim}\left(A(S) / A_{1}\right) \geq \operatorname{dim}\left(A(S) / A_{2}\right)$. By symmetry the dimensions must be equal and hence the automorphism of $A(S)$ is an isomorphism of $A_{1}$ and $A_{2}$.

The proof of Proposition 5 in [11] used the presence of homology in $M\left(A_{i}\right)$ to insure that $\tau$ is an automorphism. If $M\left(A_{1}\right)=M\left(A_{2}\right)=D$ then that proof yields a sequence of linear maps $T_{n}$ of $A_{1}$ onto $A_{2}$, homeomorphism $t_{n}$ of $\partial D$ to $\partial D$, an analytic map $\tau$ of $D \backslash \partial D$ into $D$ and a sequence of numbers $\varepsilon_{n}$ tending to zero so that for all $f$ in $A_{1}$

$$
\sup _{\partial D}\left|T_{n} f-f \circ t_{n}\right| \leq \varepsilon_{n}\|f\|
$$


and

$$
T_{n} f \rightarrow f \circ \tau
$$

uniformly on compact subsets of $D \backslash \partial D$.

Let $G$ be one of the functions in $A_{1}$ of the type described in Proposition 2.5. $G$ is a Blaschke product with $m$ zeros. Using (2.6) and the argument principle we see that $T_{n} G=B_{n} F_{n}$ with $B_{n}$ a Blaschke product with $m$ zeros and $F_{n}$ an analytic function which satisfies

$$
1-\varepsilon_{n} \leq\left|F_{n}(z)\right| \leq 1+\varepsilon_{n} \text { for all } z \text { in } D .
$$

LEMMA 2.10. Suppose $H_{n}=B_{n} F_{n}$ are functions in the disk algebra, each $B_{n}$ a Blaschke product with $m$ zeros and $F_{n}$ satisfy (2.8) for a sequence of $\varepsilon_{n}$ which tends to zero. Suppose $H_{n}$ converges uniformly on compact subsets of $D \backslash \partial D$ to a limit $H$ then either

(a) $H$ is a Blaschke product with $m$ zeros, or

(b) $H$ is a constant of modulus 1 .

If case (b) occurs, then the zeros of $B_{n}$ converge to $\partial D$ as $n$ becomes large.

Proof. Elementary function theory.

We apply the lemma to $H_{n}=T_{n} G$. By (2.7) $H_{n}$ converges to $G \circ \tau$. We now rule out the possibility described in (b) of the conclusion by showing that the zeros of $T_{n} G$ do not converge to $\partial B$. For any $F$ in the disk algebra which doesn't vanish on $\partial D$, denote by $\omega(F)$ the winding number of $F(\partial D)$ about the origin. Thus $\omega(F)$ is the size of $\left\{F^{-1}(0)\right\}$ (counting multiplicity). Suppose now that the zeros of $T_{n} G$ did go out to the boundary $\partial D$. Then, given $\varepsilon>0$ there is a neighborhood $N$ of $\partial D$ such that for any $p$ in $N \backslash \partial D$ there is an $F=F_{p}$ in $A_{2}$ with $F_{p}(p)=0$, $1-\varepsilon<\left|F_{p}\right|<1+\varepsilon$ on $\partial D$, and $\omega\left(F_{p}\right)=1$ (such $F_{p}$ can be obtained by composing the functions produced in Proposition 2.6 with automorphisms of the disk.) Suppose that for large $n$ the zeros of $T_{n} G$ are in $N$. Hence $T_{n} G=\prod_{i=1}^{m} F_{i} R$ where $F_{i}=F_{p_{i}}$ and $p_{i}$ is the $i$ th zero of $T_{n} G . \omega(R)=0$ and $|R|$ is approximately 1 on $\partial D$. Since the only zeros of $F_{i}$ are in $N$, we can apply Lemma 2.4 and conclude that $R$ is in $A$. We can thus apply $T_{n}^{-1}$ to the $F_{i}$ and to $R$

$$
G=T_{n}^{-1}\left(\left(\prod_{i=1}^{m} F_{i}\right) R\right)
$$

Using (2.6), this implies

$$
G=\left(\prod\left(T_{n}^{-1} F_{i}\right)\right) T_{n}^{-1} R+r
$$


for some remainder $r$ which has norm which is $O\left(\varepsilon_{n}\right)$. Thus, if $n$ is large then using (2.6) again, $T_{n}^{-1} R$ must have modulus approximately one on $\partial D$; and, again by $(2.6), \omega\left(T_{n}^{-1} R\right)=0$. Hence $T_{n}^{-1} R$ is an invertible element of the disk algebra. Since $\operatorname{dim} A(D) / A_{1}$ is finite, this implies $T_{n}^{-1} R$ is an invertible element of $A_{1}$. Thus

$$
(G-r)\left(T_{n}^{-1} R\right)^{-1}=\prod\left(T_{n}^{-1} F_{l}\right) .
$$

Evaluate the left-hand side of this at any point $p$ in $\operatorname{Sing}\left(A_{1}\right)$. Since $G(p)=0$ and $r$ is small, at least one of the factors $T_{n}^{-1} F_{l}$ must be small at $p$. Suppose $T_{n}^{-1} F_{1}(p)=\delta$ for some small $\delta$. The function $H=T_{n}^{-1} F_{1}-\delta$ vanishes at $p$ and at no other point of $D$ (since $\omega(H)=1) . H$ is in $A$. Hence we have found a function $H$ in $A$, with a simple zero at $p$ and no other zero in $D$. This contradicts the fact that $p$ is in $\operatorname{Sing}\left(A_{1}\right)$.

Thus $G \circ \tau$ is a Blaschke product with exactly $m$ zeros.

Lemma 2.11. Suppose $\tau$ maps $D \backslash \partial D$ analytically into $D$ and that there is a Blaschke product $G$ which has $m$ zeros and such that $G \circ \tau$ is also a Blaschke product with exactly $m$ zeros. Then $\tau$ is a conformal automorphism of the disk.

Proof. Elementary function theory.

Once we know $\tau$ is an automorphism, the proof is finished as before.

The one remaining case is $A_{1}=A_{2}=A(D)$. In that case, $\operatorname{Sing}\left(A_{i}\right)$ is empty and we can't show $\tau$ is an automorphism. In fact, in that case, $\tau$ may be a constant map. However, in that case, we still have $A_{1}=A_{2}$.

III. The Global Theory of $\mathscr{A}_{k}$. For any $A, B$ in $\mathscr{A}$; we denote by $d(A, B)$ the Banach-Mazur distance between the spaces $A$ and $B$. If $d(A, B)$ is small then $d(A, B)$ is equivalent to the smallest $\varepsilon$ so that $B$ is an $\varepsilon$-deformation of $A$ (Theorem 4.1 of [15]). We will always assume (often without mention) that the distances involved are so small that this result applies.

Suppose $A$ is in $\mathscr{A}_{k}$ and $B$ is in $\mathscr{M}$ with $d(A, B)<\varepsilon$ for some small $\varepsilon$. By Theorem 4.1 of [15], there is a $T$ in $L(A, B)$, a homeomorphism $\tau$ of $\partial B$ to $\partial A$ and a universal constant $c$ so that for all $f, g$ in $A$ all $y$ in $\partial B$

$$
\left\{\begin{array}{l}
T 1=1 \\
|T f(y)-f(\tau(y))| \leq c \varepsilon\|f\| \\
\|T(f g)-T f T g\| \leq c \varepsilon\|f\|\|g\|
\end{array}\right\} .
$$


For any algebra $A$, let $A^{-1}$ denote the invertible elements of $A . T$ also satisfies the following. Given a $K>1$, there is a $\delta=\delta(K)$ so that

$$
\text { if } a \text { is in } A^{-1},\|a\|\left\|a^{-1}\right\| \leq K, \varepsilon<\delta \text { then } T a \text { is in } B^{-1} \text {. }
$$

Let $\mathscr{S}^{*}$ be the set of finite bordered connected possibly singular surfaces which are obtained as $M(A)$ for $A$ in $\mathscr{A}$. For $S$ in $\mathscr{S}^{*}$, let $A(S)$ be the algebra of continuous functions on $S$ holomorphic on $S \backslash \partial S$.

THEOREM 3.1. Let $k$ be a non-negative integer. There are positive constants $\varepsilon_{k}$ and $c_{k}$ which depend only on $k$ so that if $A$ is in $\mathscr{A}_{k}$ and $B$ is a uniform algebra with $d(A, B)<\varepsilon<\varepsilon_{k}$ then there is an $S(B)$ in $\mathscr{S}^{*}$ such that $B$ is a subalgebra of $A(S(B)), \partial B=\partial S(B), M(B)=S(B)$. Furthermore, there is a $T$ in $L(A, B)$ and a homeomorphism $\tau$ of a neighborhood $N_{B}$ of $\partial S(B)$ onto a neighborhood $N_{A}$ of $\partial S(A)$ so that for all $f, g$ in $A$, all $y$ in $N_{B}$

$$
\left\{\begin{array}{l}
T 1=1 \\
|T f(y)-f(\tau(y))| \leq c_{k} \varepsilon\|f\| \\
\|T f g-T f T g\| \leq c_{k} \varepsilon\|f\|\|g\|
\end{array}\right\} .
$$

Furthermore, on $N_{B} \backslash \partial S(B)$ the map $\tau$ is a quasiconformal homeomorphism with maximal dilatation at most $\left(1+c_{k} \varepsilon\right)$.

Note. It is not claimed that $B$ is in $\mathscr{A}$. That is, it is not claimed that $\operatorname{dim}(A(S(B)) / B)<\infty$. We will show that this is true if further assumptions are made on $B$ or if we allow the constant $\varepsilon_{k}$ to depend on $A$.

Proof. Throughout the proof, we will use the letter $c$ for various constants which can be selected so as to only depend on $k$. We start by assuming that we have a uniform algebra $B$ and a $T$ in $L(A, B)$ which satisfies (3.1) and (3.2). The values of $\varepsilon_{k}$ and $c_{k}$ will be implicit in the proof (but will only depend on $k$ ).

Let $S=S(A)=M(A)$.

Pick and fix a function $G$ in $A$ which satisfies the conditions described in Proposition 2.5. Let $J$ be the valance of $G$. Recall that $J$ is bounded by a bound which depends only on $k$.

We now use Proposition 2.6 to construct a neighborhood of $\partial S$. Suppose we have $\eta, p, N_{1}, N_{2}$ and $F$ so that the hypotheses and conclusions of that proposition are satisfied. Suppose $N$ is another neighborhood of $p, p \in N \subseteq N_{1}$. There are $\tilde{N}_{1}, \tilde{N}_{2}, \tilde{F}$ with $p \in \tilde{N}_{1} \subset \tilde{N}_{2} \subseteq N$ so that the conclusions of the proposition are satisfied with the new neighborhoods 
$\tilde{N}_{1}, \tilde{N}_{2}$, the new function $\tilde{F}$ and the same $\eta$. This can be checked by composing $F$ with automorphisms of the disk. Hence we can shrink the neighborhoods $N_{i}$ without having to increase $\eta$. Thus we can, for fixed $\eta$ (the size of which will be implicit later), select neighborhoods $N_{1}(p)$, $N_{2}(p)$ and functions $F_{p}$ which satisfy the conclusions of Proposition 2.6 and which also satisfy, for each $p$ in $\partial S$

$$
\left(G^{-1}\left(G\left(N_{1}(p)\right)\right)\right) \backslash N_{1}(p) \cap N_{2}(p)=\varnothing .
$$

That is, if we restrict to $N_{2}(p)$ then $G$ is univalent on $N_{1}(p)$.

Let $N_{1}$ be the union of the sets $N_{1}(p)$ for $p$ in $\partial S$. Pick $\alpha$ so small that

$$
\{q \in S ; 1-2 \alpha<|G(q)| \leq 1\} \subseteq N_{1} \text {. }
$$

Let

$$
N_{A}=\{q \in S ; 1-\alpha \leq|G(q)|<1\} .
$$

We will construct a map $\sigma$ of $N_{A}$ into $M(B)$. The required map $\tau$ will be $\sigma^{-1}$.

Pick $y$ in $N_{A}$. Let $y_{1}=y$ and let $y_{2}, \ldots, y_{J}$ be the other points of $G^{-1}(G(y))$. Using (3.4) and the definition of $N_{A}$, we see that if we compose $G$ with appropriate automorphisms of the disk, then we can obtain for each $i, i=1, \ldots, J$, a function $G_{i}$ in $A$ with $\left\|G_{i}\right\|=1, G_{i}\left(y_{i}\right)=0$ and $\left|G_{i}\left(y_{j}\right)\right|<\eta$ for $j=1, \ldots, J ; j \neq i$. Select such $G_{i}$ and then, for $i=1, \ldots, J$ set

$$
H_{\imath}=\prod_{\substack{j=1 \\ j \neq i}}^{J} G_{j} .
$$

Thus $H_{i}$ is in $A$ and for $1 \leq i, j \leq J$,

$$
\left\|H_{i}\right\|=1, \quad H_{i}\left(y_{j}\right)=0, \quad i \neq j, \quad\left|H_{i}\left(y_{\imath}\right)-1\right|<c \eta .
$$

Let $F=F_{y}=(G-G(y))(1-\overline{G(y)} G)^{-1}$. Thus $F$ is in $A,|F|=1$ on $\partial A$ and $F^{-1}(0)=\left\{y_{1}, \ldots, y_{n}\right\}$.

Claim. $(T F)^{-1}(0)$ consists of $J$ points, $x_{1}, \ldots, x_{J}$. There is a unique numbering of these points so that if we set $\sigma\left(y_{i}\right)=x_{i}$ (i.e., $\tau\left(x_{i}\right)=y_{i}$ ) then (3.3) is satisfied.

Proof of the claim. First note that if $f$ is in $A$ then

$$
f=\sum a_{i} H_{\imath}+F r
$$

for scalars $a_{i}$ and an $r$ in $A$ which satisfies

$$
\|r\| \leq(2 J+1)\|f\| \text {. }
$$

This estimate is an immediate consequence of the explicit choice $a_{\imath}=$ $f\left(y_{i}\right) / H_{l}\left(y_{l}\right)$ and (3.5). 
(3.6) and (3.7) express the fact that we can select a complement of the ideal $(G-G(y)) A$ in such a way that the norm of the projection onto this complement doesn't depend on $y$ for $y$ in $N_{A}$ (nor on $A$ ). This uniformity is one of the factors in obtaining the uniform constants in the theorem.

If $\varepsilon$ is small, then the ideal $(T F) B$ inherits this property. That is, for each $g$ in $B$ there are scalars $b_{i}$ and a function $r^{\prime}$ in $B$ so that

$$
g=\sum b_{i} T H_{i}+(T F) r^{\prime}
$$

and

$$
\left|b_{i}\right| \leq c\|g\|, \quad i=1, \ldots, J ; \quad\left\|r^{\prime}\right\| \leq c\|g\| .
$$

Furthermore, the $b_{i}$ and $r^{\prime}$ are uniquely determined. These facts are straightforward consequences of (3.1), (3.6) and (3.7).

Let $E$ be the algebra of complex valued functions on the set $\left\{y_{1}, \ldots, y_{J}\right\}$. For $s$ in $E$ set $\|s\|=\max \left|s\left(y_{i}\right)\right|$. Define $L$ mapping $E$ to $H=\operatorname{Span}\left\{H_{1}, \ldots, H_{J}\right\}$ by

$$
L s=\sum_{i} s\left(y_{i}\right) H_{i} / H_{i}\left(y_{i}\right) .
$$

By (3.5), we have,

$$
\|s\| \mid\|L s\| \leq c\|\| s \| .
$$

We now define a new multiplication, $\times$, on $E$ by

$$
s \times t=r
$$

where $r$ satisfies

$$
(T L s)(T L t)=(T L r)+(T F) b
$$

for some $b$ in $B$. Since $T L r$ is in $T H$ we can use (3.9) and (3.10) to obtain

$$
\|b\| \leq c\|\| s\|\mid\| t\|\| \text {. }
$$

We now estimate the difference between the two multiplications on $E$. By (3.1), (3.10) and (3.11)

$$
\begin{aligned}
\|s \times t-s t\| & \leq c\|L(s \times t)-L(s t)\| \\
& \leq c\left\|T^{-1}((T L s)(T L t)-(T F) b)-L(s t)\right\| \\
& \leq c\left\|L s L t-L(s t)-F T^{-1} b+a_{1}+a_{2}\right\|
\end{aligned}
$$

where

$$
\begin{aligned}
& a_{1}=T^{-1}((T L s)(T L t))-L s L t \\
& a_{2}=-T^{-1}((T F) b)+F T^{-1} b
\end{aligned}
$$


by (3.1) and (3.12), for $i=1,2$,

$$
\left\|a_{i}\right\| \leq c \varepsilon|\|s|\||\|t \mid\| .
$$

The quantity in the norm sign on the right-hand side of (3.13) is in $H$. Hence, by (3.10) we can estimate its norm by evaluating at the points $y_{i}$. At each of the $y_{i}, L s t=L s L t$ and $F$ is zero. Hence

$$
\|s \times t-s t\| \leq c\left\|a_{1}+a_{2}\right\| .
$$

When this is combined with (3.14) we obtain

$$
\|s \times t-s t|\|\leq c \varepsilon\| s|\||\|t \mid\| .
$$

Now note that $T L$ is an algebra map of the algebra $(E, \times)$ to the quotient algebra $B /(T F) B$. (In particular, $\times$ is an associative product.) Hence, if $\varepsilon$ is sufficiently small we can use Theorem 3.1 of [15] (which described small deformations of uniform algebras). That theorem insures that the spectrum of $E$ is homeomorphic to the spectrum of $B /(T F) B$. Thus $\left\{(T F)^{-1}(0)\right\}$ has $J$ points. Furthermore, that theorem insures that there is a mapping $\sigma$ of the $y_{i}$ to $\sigma\left(y_{i}\right)$ in $M(B /(T F) B)$ so that

$$
\left\|L s\left(y_{i}\right)-(T L s)\left(\sigma\left(y_{i}\right)\right)\right\| \leq c \varepsilon\|\mid s\|
$$

for all $s$ in $E$. Write $x_{i}$ for $\sigma\left(y_{l}\right)$. Combining (3.16) with the earlier estimates (3.1), (3.6), (3.7) and (3.9) and (3.10) shows

$$
\left\|f\left(y_{\imath}\right)-(T F)\left(\sigma\left(y_{i}\right)\right)\right\| \leq c \varepsilon\|f\|
$$

for all $f$ in $A$. Finally note that elementary estimates using (3.16) and (3.5) show that the choice of $\sigma$ is uniquely determined. This completes the proof of the claim.

The proof was carried out in the language of the deformation theory of the uniform algebra $E$. However, the proof actually used the norm equivalence of $E$ with the quotient algebra $A / F A$ (which is a consequence of the finite dimensionality of $A / F A$ and is uniform by virtue of (3.5)) and the fact that the quotient algebra $B /(T F) B$ is a small deformation of $A / F A$. It would be interesting to have a deformation theory for such quotient algebras. Also, we point out that we could have given a direct proof of the claim (not using results of [15]). That proof would have been a bit longer.

The construction of $\sigma(y)$ used the particular function $F_{y}$. We now extend the definition of $\sigma$ to all $N_{A}$ by using the same construction and letting $y$ vary over $N_{A}$. The functions $H_{i}$ with the properties described in (3.5) will then depend on the base point $y$. We will not, however, explicitly exhibit this dependence. 
For each fixed $y$ in $N_{A}$, we have shown that $\operatorname{card}\left\{(T F)^{-1}(0)\right\}=$ $\operatorname{dim} B /(T F) B<\infty$. This is sufficient to conclude that $(T F)^{-1}\{z ;|z|<.9\}$ can be given the structure of an open (possibly disconnected) Riemann surface with point identifications and on which the functions in $B$ are analytic (VI 6.3 of [2]). Next note that the proof of the claim extends immediately to show that if $|\delta|<.1$ then $(T F)^{-1}(\delta)$ consists of $J$ points. Hence the Riemann surface has neither ramification nor point identification over $\{z ;|z|<.1\}$. By (3.3) and (3.5), the functions $T F$ and $T H_{t}$ separate the components of $(T F)^{-1}\{z ;|z|<1$.$\} from each other and from$ the rest of $M(B)$. Hence those functions completely determine the topology and conformal structure of $M(B)$ near $\left\{(T F)^{-1}(0)\right\}$.

We now show that $\sigma$ is continuous. Suppose $z_{n}$ is a sequence converging to $y$. The function $F_{\zeta}$ depends in a norm continuous way on $\zeta$; hence $\left|T F_{y}\left(\sigma\left(z_{n}\right)\right)-T F_{z_{n}}\left(\sigma\left(z_{n}\right)\right)\right|$ tends to zero. Since $T F_{z_{n}}\left(\sigma\left(z_{n}\right)\right)=0$ by definition, we have, for any $\delta>0, \sigma\left(z_{n}\right) \in(T F)^{-1}\{z ;|z|<\delta\}$ for all large $n$. To see that no subsequence of $\sigma\left(z_{n}\right)$ can converge to a point of $\left\{(T F)^{-1}(0)\right\}$ other than $y$, note that by

$$
\left|T H\left(\sigma\left(z_{n}\right)\right)-H\left(z_{n}\right)\right| \leq c \varepsilon\|H\|
$$

for all $H$ in $A$. Hence, by (3.3) and (3.5), all of the $\sigma\left(z_{n}\right)$ are in the sheet of $(T F)^{-1}\{z:|z|<\delta\}$ on which a particular $T H_{l}$ is small and all the others are large.

We now show that $\sigma\left(N_{A}\right) \cup \partial B$ contains an open neighborhood of $\partial B$. If not, we could find a sequence $\left\{x_{n}\right\}$ of points in $M(B) \backslash \partial B$ which converges to some $x_{\infty}$ in $\partial B$. Without loss of generality $G\left(\tau\left(x_{\infty}\right)\right)=1$. For $\lambda$ real and slightly less than one, $G_{\lambda}=(G-\lambda)(1-\lambda G)^{-1}$ is one of the functions $F_{y}$ for $y$ in $N_{A}$. Also, $G_{\lambda}\left(\tau\left(x_{\infty}\right)\right)=1$. By (3.1), $\left|T G_{\lambda}\left(x_{\infty}\right)-1\right|$ $<c \varepsilon$. Since $x_{n} \rightarrow x_{\infty},\left|T G_{\lambda}\left(x_{n}\right)-1\right|<2 c \varepsilon$ for all $n$ larger than some $N_{0}$. Pick $x=x_{N_{0+1}}$ and let $\mu$ be a measure on $\partial B$ which is a representing measure for $x$. Let $\nu=\mu \circ \tau^{-1}$ (i.e., $\nu(R)=\mu\left(\tau^{-1}(R)\right)$ for $\left.R \subseteq \partial A\right)$. Since every point of $\partial B$ is a strong peak point for $B$ (Theorem 3.1 of [15]), $\mu$ has no atoms. Hence we can select a unique point $Q$ on $\partial D$ so that if $\Gamma$ is the arc counterclockwise from $Q$ to -1 then $\nu\left(G_{\lambda}^{-1}(\Gamma)\right)=1 / 2$. Also note that $Q$ is very close to one. This can be seen by the following calculation:

$$
\begin{aligned}
1-2 \varepsilon & \leq \operatorname{Re} T G_{\lambda}(x)=\operatorname{Re} \int\left(T G_{\lambda}\right) d \mu \\
& =\operatorname{Re} \int_{\partial A}\left(T G_{\lambda}\right) \circ \tau^{-1} d \nu=\operatorname{Re} \int_{\partial A} G_{\lambda} d \nu+\varepsilon_{1}
\end{aligned}
$$


where, by (3.1), the error $\varepsilon_{1}$ has size $O(\varepsilon)$. Hence

$$
\begin{aligned}
1-c \varepsilon & \leq \operatorname{Re} \int_{\partial A} G_{\lambda} d \nu=\operatorname{Re}\left(\int_{G_{\lambda}^{-1}(\Gamma)}+\int_{\partial A \backslash G_{\lambda}^{-1}(\Gamma)}\right) \\
& \leq \frac{1}{2} \operatorname{Max}\left\{\operatorname{Re} G_{\lambda}(p) ; G_{\lambda}(p) \in \Gamma\right\}+\frac{1}{2} \operatorname{Max}\left\{\operatorname{Re} G_{\lambda}\right\} \\
& \leq \frac{1}{2} \operatorname{Re} Q+\frac{1}{2} .
\end{aligned}
$$

Thus $\operatorname{Re} Q>1-c \varepsilon$.

Let $L$ be the automorphism of $D$ which fixed -1 and takes $Q$ to 1 . An elementary calculation shows that for $0 \leq \delta<1$, the functions

$$
F_{\delta}=\left(L\left(G_{\lambda}\right)-\delta\right)\left(1-\delta L\left(G_{\lambda}\right)\right)^{-1}
$$

are among the functions $F_{y}$ for $y$ in $N_{A}$.

We now claim that for some $\delta, 0 \leq \delta<1,\left|T F_{\delta}(x)\right|<2 / 3$. First note that $G_{\lambda}^{-1}(\Gamma)$ is a set of $\nu$ measure $1 / 2$ on which $\operatorname{Im} F_{\delta}$ is positive. On the complementary set $\operatorname{Im} F_{\delta}$ is negative. Hence, by (3.1) and the fact that $\nu$ represents $x$,

$$
\left|\operatorname{Im} T F_{\delta}(x)\right| \leq .6
$$

for all $\delta$. Now note that $\operatorname{Re} T F_{\delta}(x)$ is a continuous function of $\delta$ and is positive when $\delta=0$. Since $\nu$ has no atoms, it is easy to check using (3.1) that $\operatorname{Re} T F_{\delta}(x)$ is negative for $\delta$ near 1 . The intermediate value of $\delta$ for which $\operatorname{Re} T F_{\delta}(x)=0$ gives the required $\delta$. We now denote that $F_{\delta}$ by $F$.

Now note that $F(\{z ;|G(z)|=1-\alpha\})$ is a small set near the point -1. $G_{\lambda}$ had that property for $\lambda$ near 1 and the passage from $G_{\lambda}=F_{0}$ to $F_{\delta}$ for positive $\delta$ makes the situation better. Hence $F^{-1}(\{z ;|z| \leq .8\})$ consists of $J$ disjoint closed homeomorphic images of the unit disk, $D_{1}, \ldots, D_{J}$, and all the $D_{i}$ are inside $N_{A}$. Pick one such $D_{i}$. On $D_{i}$ there is defined a continuous function $R(z)=T F(\sigma(z))$. Since $|R(z)-F(z)| \leq c \varepsilon, R(z)$ maps $\partial D_{i}$ onto a curve which winds once about zero. Hence, using standard considerations from algebraic topology, we find that there is a point $z_{i}$ in $D_{i}$ at which $R$ takes the value $T F_{\delta}(x)$. Thus $T F\left(\sigma\left(z_{i}\right)\right)=T F_{\delta}(x)$ for some $z_{i}$ in $D_{i}$. This accounts for $J$ distinct points of $T F^{-1}\left(T F_{\delta}(x)\right)$. However we saw earlier that $\operatorname{card}\left\{T F^{-1}\left(T F_{\delta}(x)\right)\right\}=J$. Thus $x$ is $\sigma(z)$ for some $z$ in $N_{A}$. This contradiction shows that $\sigma\left(N_{A}\right) \cup \partial B$ contains a neighborhood of $\partial B$.

We now extend $\sigma$ from $N_{A}$ to $\overline{N_{A}}$. On the set $\{z ;|G(z)|=1-\alpha\}$ we use the same definition. On $\partial S=\{z ;|G(z)|=1\}$ we set $\sigma=\tau^{-1}$ where $\tau$ is described by (3.1). We now claim that this extended map is continuous. Since $\tau$ is a homeomorphism of $\partial B$ to $\partial S$, the only real issue is to show 
that if $y_{n}$ are in $S \backslash \partial S$ and $y_{n} \rightarrow y_{\infty}$ in $\partial S$ then $\sigma\left(y_{n}\right) \rightarrow \sigma\left(y_{\infty}\right)$. Suppose we are given such $y_{n}$ and that $x$ is an accumulation point of the points $\sigma\left(y_{n}\right)$. If $x$ is not in $\partial B$ then the argument used in the previous two paragraphs can be used to show that there is some $F_{y}$ with $\left|T F_{y}(x)\right|<2 / 3$. However,

$$
\left|T F_{y}(x)\right| \geq \underline{\lim }\left|T F_{y}\left(\sigma\left(y_{n}\right)\right)\right| \geq \underline{\lim }\left|F_{y}\left(y_{n}\right)\right|-c \varepsilon=1-\varepsilon .
$$

Thus $x$ is in $\partial B$. Thus $\sigma\left(y_{n}\right) \rightarrow \sigma(\bar{y})$ for some $\bar{y}$ in $\partial A$. If $y_{\infty} \neq \bar{y}$ then there is an $H$ in $A$ with $\|H\|=1, H\left(y_{\infty}\right)=1$ and $H(\bar{y})=0$. Thus $T H(x)=$ $T H(\sigma(\bar{y})) \sim H(\tau(\sigma(\bar{y})))=H(\bar{y})=0$ (by (3.1)). On the other hand $T H(x)=\lim T H\left(\sigma\left(y_{n}\right)\right)$ and $T H\left(\sigma\left(y_{n}\right)\right) \sim H\left(y_{n}\right)$ which converges to $H\left(y_{\infty}\right)=1$. This contradiction shows $y_{\infty}=\bar{y}$ and we conclude $\sigma$ is continuous on $\overline{N_{A}}$.

We now show $M(B) \backslash \partial B$ is a Riemann surface. Select a large integer $N$ so that $(1-\alpha)^{N} \leq 1 / 2$. Consider the map of $M(B)$ into $\{z ;|z|<1+$ $c \varepsilon\}$ given by $R=T\left(G^{N}\right)$. Note that $\sigma\left(\bar{N}_{A}\right)$ is a closed subset of $M(B)$ which contains an open neighborhood of $\partial B$. Let $U=M(B) \backslash \sigma\left(\overline{N_{A}}\right)$. By the local maximum modulus theorem, for all $x$ in $U$, all $f$ in $B$

$$
|f(x)| \leq \max _{\partial U}|f| \text {. }
$$

Now note that $\partial U \subseteq \sigma(\{z \in M(A) ;|G(z)|=1-\alpha\})$. Hence, for $x$ in $U$,

$$
|R(x)| \leq \sup \left\{\left|T\left(G^{N}\right)(\sigma(x))\right| ;|G(x)|=1-\alpha\right\} .
$$

Thus, by (3.3) and the choice of $N$, for all $x$ in $U$,

$$
|R(x)| \leq 1 / 2+c \varepsilon
$$

Note that for $x$ in $\partial B$

$$
|R(x)| \geq 1-c \varepsilon .
$$

We now claim that for each $z$ in a small neighborhood of $3 / 4,\left\{R^{-1}(z)\right\}$ is a finite set. Suppose not and let $x_{0}$ be an accumulation point of $\left\{R^{-1}(z)\right\}$ for such a $z$. By (3.17) and (3.18), it must be that $x_{0}$ is in $\sigma\left(N_{A}\right)$. Hence there is a neighborhood of $x_{0}$ which is an analytic disk. Hence $R$ takes the value $z$ identically in an open neighorhood of $x_{0}$. In this case the interior of $\left\{R^{-1}(z)\right\}$ would be non-empty, open, and, by the argument just given, closed. Any such set must meet the Shilov boundary (Corollary 8.16 of [17]) and by (3.18) this doesn't meet $\partial B$. Thus $\left\{R^{-1}(z)\right\}$ is finite.

Thus we have an open set of $z$ for which $\operatorname{card}\left\{R^{-1}(z)\right\}$ is finite. Hence we may use a result of Bishop (Theorem 11.2 of [18]) to conclude that for each with $|z|<1-c \varepsilon,\left\{R^{-1}(z)\right\}$ has a neighborhood which 
consists of a finite union of analytic disks. Thus $U$ is an open (possibly disconnected) Riemann surface which might have finitely many point identifications. Also each point of $\sigma\left(N_{A}\right)$ has a neighborhood which is an analytic disk. Thus $M(B) \backslash \partial B$ is a finite Rieman surface.

We have not yet shown that $M(B)$ is a finite bordered Riemann surface. To do this we must exhibit neighborhoods of the appropriate form for the points of $\partial B$. This will follow immediately from the corresponding facts for $M(A)$ as soon as we know that $\sigma$ is a homeomorphism onto its image. We already know $\sigma$ is continuous. Hence we need to verify the following.

Claim. $\sigma$ is one-to-one on $N_{A}$.

Proof of the claim. Suppose $\sigma\left(y_{1}\right)=\sigma\left(y_{2}\right)$. By (3.3) $T F_{y_{1}}\left(\sigma\left(y_{1}\right)\right)=$ $T F_{y_{1}}\left(\sigma\left(y_{2}\right)\right) \sim F_{y_{1}}\left(y_{2}\right)$. Hence $y_{2}$ is in $F_{y_{1}}^{-1}(\{|z|<.1\})$. That set consists of $J$ disjoint analytic disks. By using the estimates in (3.3) and (3.5) we see that $y_{2}$ and $y_{1}$ must be in the same disk. On that disk $F=F_{y_{1}}$ is a coordinate function. In terms of this $F$, the function $F_{y_{2}}$ used to define $\sigma\left(y_{2}\right)$ is of the form $F_{y_{2}}=(F-\delta)(1-\bar{\delta} F)^{-1}$ with

$$
F\left(y_{2}\right)=\delta, \quad|\delta|<c \varepsilon .
$$

Thus $F_{y_{2}}=F-\delta+\bar{\delta} F^{2}+O\left(|\delta|^{2}\right)$. If we apply $T$ we obtain

$$
T F_{y_{2}}=T F-\delta+\bar{\delta} T\left(F^{2}\right)+O\left(|\delta|^{2}\right) .
$$

We evaluate this at $\sigma\left(y_{2}\right)$. By definition $T F_{y_{2}}\left(\sigma\left(y_{2}\right)\right)=0$. We assumed $\sigma\left(y_{1}\right)=\sigma\left(y_{2}\right)$. Thus $T F\left(\sigma\left(y_{2}\right)\right)=0$. By (3.1), $T\left(F^{2}\right)=(T F)^{2}+O(\varepsilon)$. Hence $T\left(F^{2}\right)\left(\sigma\left(y_{2}\right)\right)=T\left(F^{2}\right)\left(\sigma\left(y_{1}\right)\right)=O(\varepsilon)$. Thus (3.19) yields

$$
\delta=\bar{\delta} O(\varepsilon)+O\left(|\delta|^{2}\right) \text {. }
$$

Sinc $|\delta|<c \varepsilon$, this implies $\delta=0$. Thus $F\left(y_{2}\right)=0$ and hence $y_{2}=y_{1}$. This proves the claim.

It only remains to show that the Riemann surface $M(B) \backslash \partial B$ is connected. By Theorem 6.2 of [15], $M(B)$ is connected. Any union of components of $M(B) \backslash \partial B$ together with the appropriate components of $\partial B$ would contain a connected component of $M(B)$. Thus $M(B) \backslash \partial B$ is connected.

COROLlary 3.2. B is antisymmetric, (i.e., the only real valued functions in $B$ are constant).

Proof. $B$ is a subalgebra of the algebra of homomorphic functions on a connected Riemann surface. 
This last corollary is noteworthy since it is not known that a small deformation of an antisymmetric uniform algebra is necessarily antisymmetric.

With further assumptions, we can show $B$ is in $\mathscr{A}$.

THEOREM 3.3. Let $k$ be a non-negative integer. There is an $\varepsilon_{k}>0$ which depends only on $k$ such that if $A$ is in $\mathscr{A}_{k}$ and $B$ is a uniform algebra with $d(A, B)<\varepsilon_{k}$ and $\operatorname{dim} \operatorname{Re} B^{\perp}<\infty$ then $B$ is in $\mathscr{A}$.

Proof. Suppose $d(A, B)<\varepsilon$. By requiring $\varepsilon$ to be small we can insure that Theorem 3.1 can be applied. Let $T$ be the element of $L(A, B)$ and $\tau$ the homeomorphism of $\partial B$ to $\partial A$ which exist by Theorem 3.1 and which satisfy (3.1) and (3.3).

Let $S=M(B)$ be the finite bordered singular Riemann surface on which the functions in $B$ are defined. Let $S_{i}, i=1, \ldots, n_{B}$, be the elements of $\mathscr{S}$ from which $S$ is constructed by point identifications. For $i=1, \ldots, n_{B}$ select $p_{\imath}$ in $S_{i}$ so that $p_{\imath}$ is not one of the points which are involved in the later point identifications. Hence the points $p_{i}$ can also be regarded as points of $S$.

A positive measure $\nu$ on $\partial B$ is a representing measure for $p$ if for all $f$ in $B$

$$
f(p)=\int_{\partial B} f d \nu
$$

We denote the set of all such measures by $M_{p}$. Since the difference of two elements of $M_{p}$ is in $\operatorname{Re} B^{\perp}$, and we have assumed $\operatorname{dim} \operatorname{Re} B^{\perp}<\infty$, the set $M_{p}$ is finite dimensional for any $p$ in $S$. We now use the terminology and some of the results of Chapter IV of the book by Gamelin [2]. For $i=1, \ldots, n_{B}$ we select a core measure $\nu_{i}$ in $M_{p_{i}}$. For our purposes this means that if $\tilde{\nu}_{i}$ is any other element of $M_{p_{t}}$ then

$$
\tilde{\nu}_{l} \ll \nu_{l}
$$

(This by Theorem 4.1 of Chapter IV of [2].) This choice will also allow us to use Theorem 6.2 of Chapter IV of [2] which describes the "nice case" of abstract Hardy space theory.

Let $B^{\perp}$ be the space of measures on $\partial B$ which are orthogonal to $B$.

Let $\nu_{0}=\sum \nu_{i}$.

LEMmA 3.4. If $\mu \in B^{\perp}$ then $\mu \ll \nu_{0}$.

Proof of the Lemma. Let $q$ be a point of $S(A) \backslash \partial A$ and let $\lambda$ be a measure on $\partial A$ which represents $q$ for all $g$ in $A$. Let $\nu$ be a measure in $M_{p_{t}}$ which represents $p_{1}$ for $A\left(S_{1}\right)$; i.e., so that $\int f d \nu=f\left(p_{1}\right)$ for all $f$ in $A\left(S_{1}\right)$. 
We start by showing that $\tau^{-1}$ is an absolutely continuous map of $(\partial A, \lambda)$ to $(\partial B, \nu)$.

Let $G$ be a function in $A$ of the type described in Proposition 2.5. Suppose $\tau^{-1}$ is not absolutely continuous. Then there is a set $E$ with $\lambda(E)=0$ and $\nu\left(\tau^{-1}(E)\right)>0$. The mapping $G$ is a finite to one mapping of $\partial A$ to $\partial D$. Furthermore, the measure obtained on $\partial A$ by using $G$ to pull $d \theta$ back to $A$ is mutually boundedly absolutely continuous with respect to $\lambda$. Hence we can find $E_{i} \subseteq \hat{E}, i=1,2$, so that

$$
\begin{gathered}
G\left(E_{1}\right) \cap G\left(E_{2}\right)=\varnothing \\
\int_{\partial D} \chi_{G\left(E_{1}\right)} d \theta=\int_{\partial D} \chi_{G\left(E_{2}\right)} d \theta=0
\end{gathered}
$$

and

$$
\nu\left(\tau^{-1}\left(E_{i}\right)\right)>0, \quad i=1,2 .
$$

Since the $G\left(E_{i}\right)$ are closed subsets of $\partial D$ of Lebesgue measure zero, they are peak interpolation sets for the disk algebra. Since the composition of $G$ with an element of the disk algebra is an element of $A$, the sets $E_{i}$ are peak interpolation sets for $A$. Using this fact and (3.1) we now construct a function in $B$ which vanishes on $\tau^{-1}\left(E_{i}\right)$ and doesn't vanish identically. Start with $F$ in $A$ which has $\left.F\right|_{E_{1}}=0,\left.F\right|_{E_{2}}=1$ and $\|F\|=1$. TF is almost the required function. By (3.1)

$$
\begin{aligned}
& \text { on } E_{1},\left|T F\left(\tau^{-1}(x)\right)\right| \leq c \varepsilon \\
& \text { on } E_{2},\left|1-T F\left(\tau^{-1}(x)\right)\right| \leq c \varepsilon .
\end{aligned}
$$

We now construct a convergent infinite series of corrections. For $i=$ $1,2, \ldots$ define $g_{i}$ by the requirements that $g_{i}$ be in $A$, for $x$ in $E_{1}$

$$
g_{i}(x)=-T\left(F-\sum_{j=1}^{i-1} g_{i}\right)\left(\tau^{-1}(x)\right)
$$

and for $x$ in $E_{2}$

$$
g_{i}(x)=1-T\left(F-\sum_{j=1}^{i-1} g_{j}\right)\left(\tau^{-1}(x)\right)
$$

and $\left\|g_{i}\right\|=\sup _{E_{1} \cup E_{2}}\left|g_{i}(x)\right|$. Since the $E_{l}$ are peak interpolation sets for $A$, such $g_{i}$ can be found. A straightforward induction starting with (3.21) and (3.22) and using (3.1) shows that $\left\|g_{n}\right\|=O\left((c \varepsilon)^{n}\right)$. Hence the function $\hat{F}=F+\sum_{l=1}^{\infty} g_{l}$ is in $A$. By construction $T \hat{F}$ vanishes identically on $\tau^{-1}\left(E_{1}\right)$ and is identically one on $\tau^{-1}\left(E_{2}\right)$. We now claim that this is 
impossible. The representing measures for $A\left(S_{1}\right)$ have the property that if a function in $A\left(S_{1}\right)$ vanishes on a set of positive measure with respect to such a measure, then it must vanish identically. Hence $\tau^{-1}$ is absolutely continuous as a map of $(\partial A, \lambda)$ to $(\partial B, \nu)$.

Now note that if we had selected a $\nu$ which represents $p_{j}$ for all $f$ in $A\left(S_{j}\right)$ we would have obtained the same conclusion.

Select $\mu$ in $B^{\perp}$. The abstract F. and M. Riesz Theorem (Theorem 23.6 of [17]) insures that $\mu$ can be written as

$$
\mu=\mu_{s}+\sum \mu_{n}
$$

where there are points $q_{n}$ in distinct Gleason parts of $\mathscr{M}(B)$ and representing measures $\sigma_{n}$ for $q_{n}$ so that $\mu_{n} \ll \sigma_{n}$ and $\mu_{s}$ is singular with respect to any representing measure for any point of $M(B)$, and $\mu_{s}, \mu_{1}, \mu_{2}, \ldots$ are all in $B^{\perp}$. The Gleason parts of $M(B)$ are the single points of $\partial B$ and the single part $M(B) \backslash \partial B$. Hence (3.23) becomes

$$
\mu=\mu_{s}+\mu_{1}
$$

with $\mu_{s} \perp \nu_{0}$ and $\mu_{1}$ is absolutely continuous with respect to $\sigma$ for some $\sigma$ in some $M_{p}$ for some $p$ in $M(B) \backslash \partial B$. Since $\sigma \ll \nu_{0}$ (by (3.20)), we have $\mu_{1} \ll \nu_{0}$. Thus to complete the proof we must show that there are no measures in $B^{\perp}$ which are singular with respect to $\nu_{0}$. Suppose $\alpha$ were such a measure, $\|\alpha\|=1$. Consider the linear function on $C(\partial A)$, the space of continuous functions of $\partial A$, given by

$$
l(f)=\int_{\partial B} f(\tau(x)) d \alpha(x) .
$$

The norm of $l$ on $A$ is small. If $f$ is in $A$ then

$$
\begin{aligned}
|l(f)| & =\left|\int f(\tau(x)) d \alpha(x)\right| \\
& \leq \int|T f-F \circ \tau| d|\alpha|+\left|\int T f d \alpha\right| .
\end{aligned}
$$

The first term is at most $c \varepsilon\|f\|$ (by (3.1)) and the second is zero since $\alpha$ is in $B^{\perp}$. Thus, by the Hahn-Banach Theorem, we may select a measure $\beta$ on $\partial A$ so that

$$
l(f)=\int_{\partial A} f d \beta
$$

for all $f$ in $A$ and $\|\beta\| \leq \varepsilon$. The measure $\alpha \circ \tau-\beta$ is orthogonal to $A$. Any measure on $\partial A$ which is orthogonal to $A$ is absolutely continuous with respect to $\lambda$. Hence,

$$
\alpha \circ \tau-\beta=h \lambda
$$


for some $h$ in $L^{1}(\partial A, d \lambda)$. We now use $\tau^{-1}$ to pull (3.24) to $\partial B$.

$$
\alpha-\beta \circ \tau^{-1}=(h \lambda) \circ \tau^{-1} \text {. }
$$

Since $\tau^{-1}$ is absolutely continuous as a map from $(\partial A, \lambda)$ to $(\partial B, \nu)$

$$
(h \lambda) \circ \tau^{-1}=\left(h \circ \tau^{-1}\right) \frac{d\left(\lambda \circ \tau^{-1}\right)}{d \nu} d \nu .
$$

Combining the last two equations we find that

$$
\alpha-\beta \circ \tau^{-1}=g d \nu
$$

for some $g$ in $L^{1}(\partial B, d \nu)$. We now take the Lebesgue decompositon of this equation with respect to $\nu_{0}$. By hypothesis $\alpha$ is singular with respect to $\nu_{0}$. $g d \nu \ll d \nu_{0}$. Hence $\alpha=\left(\beta \circ \tau^{-1}\right)_{\text {sing }}$. Thus

$$
1=\|\alpha\|=\left\|\left(\beta \circ \tau^{-1}\right)_{\text {sing }}\right\| \leq\left\|\beta \circ \tau^{-1}\right\|=\|\beta\| \leq c \varepsilon .
$$

This contradiction completes the proof of the lemma.

We now use abstract Hardy space theory to complete the proof of the theorem. Let $\hat{B}=A\left(S_{1}\right) \oplus \cdots \oplus A\left(S_{n_{B}}\right)$. We must show $\operatorname{dim} \hat{B} / B<\infty$. It suffices to show that there is a finite dimensional subspace $M$ of $C(\partial B)^{*}$ so that

$$
B^{\perp} \subseteq \hat{B}^{\perp}+M .
$$

Take a functional in $B^{\perp}$. By the lemma this functional can be realized as integration against an $f$ in $L^{1}\left(d \nu_{0}\right)$.

Let $\sigma_{i}$ be measures on $S_{i}$ which represent $p_{l}$ for the algebra $A\left(S_{i}\right)$ (and hence also for $B$ ). Then

$$
A_{0}\left(S_{i}\right)^{\perp}=H^{1}\left(d \sigma_{l}\right)+M_{i}
$$

where $A_{0}\left(S_{i}\right)$ is the subspace of $A\left(S_{i}\right)$ of functions which vanish at $p_{i}$ and $H^{1}\left(d \sigma_{i}\right)$ is the closure of $A\left(S_{i}\right)$ in $L^{1}\left(\partial S_{i}, d \sigma_{i}\right)$ and $M_{i}$ is a finite dimensional set of functions defined on $\partial S_{i}$. Since

$$
\hat{B}^{\perp}=\bigcap A\left(S_{i}\right)^{\perp} \text {. }
$$

We can establish (3.25) by showing that for each $i$ there is a finite dimensional set of functions $N_{i}$ which are supported on $\partial S_{i}$ and so that given $f$ in $B^{\perp}$ there is, for each $i$, an $n_{i}$ in $N_{i}$ so that $f-n_{i} \in A\left(S_{l}\right)^{\perp}$. By (3.26) this will be established if we show that we could select $n_{i}$ so that $f-n_{i}$ is in $H^{1}\left(d \sigma_{i}\right)$. We know $f$ is in $L^{1}\left(d \nu_{0}\right)$. Hence it is in $L^{1}\left(d \nu_{i}\right)$. By Theorem 4.5 of Chapter IV of [2], we must have $f=f_{1}+n$ where $f_{1}$ is in the closure of $B$ in $L^{1}\left(d \nu_{i}\right)$ and $n$ is in the complexification of the finite dimensional space $\operatorname{Re} B^{\perp}$. Since $\nu_{i}$ is a core measure for the algebra $B$ and 
$\sigma_{i}$ represents $p_{i}$ for $B$, we have (Chapter IV, Theorem 5.1 of [2]) $d \sigma_{i} / d \nu_{i}<c$ for some constant $c$. Hence $f-n$ is in the $L^{1}\left(d \sigma_{i}\right)$ closure of $B$. Hence $f-\left(n_{l} \chi_{\partial S_{t}}\right)=f-n_{l}$ is the required function in $H^{1}\left(d \sigma_{i}\right)$. The proof is complete.

Hence, $\mathscr{A}_{k}$ is open.

Corollary 3.5. Given $A$ in $\mathscr{A}_{k}$ there is an $\varepsilon(A)>0$ so that if $B$ is a uniform algebra with $d(A, B)<\varepsilon(A)$ then $B$ is in $\mathscr{A}_{k}$.

Proof. This follows from the previous Theorem and Theorem 6.3 of [15].

Also $\mathscr{A}_{k}$ is complete (and in particular closed).

COROLlaRY 3.6. Each $\mathscr{A}_{k}$ is a closed subset of $\mathscr{M}$ and a complete metric space.

Proof. $\mathscr{A}_{k} \subseteq \mathscr{M}$ and it is shown in [15] that $\mathscr{M}$ is complete. Thus we need to show $\mathscr{A}_{k}$ is closed. Let $A$ be in $\overline{\mathscr{A}}_{k}$. By the continuity on $\mathscr{M}$ of the function $\operatorname{dim} \operatorname{Re}()^{\perp}$, we know $\operatorname{dim} \operatorname{Re} A^{\perp}=k$. Hence we can apply the previous theorem to see that $A$ is in $\mathscr{A}$ and hence in $\mathscr{A}_{k}$.

Question. We just saw $\mathscr{A}_{k}=\overline{\mathscr{A}}_{k}$. Is $\mathscr{A}=\overline{\mathscr{A}}$ ? The results in [15] leave open the possibility that there is a sequence $A_{j}$ in $\mathscr{A}_{k_{j}}$ which is a Cauchy sequence in $\mathscr{M}$ and for which $\lim _{j} k_{j}=\infty$. For example, it $A_{j}=\{f \in$ $\left.A(D) ; f^{(n)}(0)=0, n=1,2, \ldots, j\right\} . A_{j}$ is in $\mathscr{A}_{2 j}$. I don't know how to show that $A_{j}$ is not a Cauchy sequence in $\mathscr{M}$.

IV. The Local Theory of $\mathscr{A}_{k}$. Corollary 3.5 states that if $A$ is in some $\mathscr{A}_{k}$ and $B$ is sufficiently close to $A$ then $B$ is in the same $\mathscr{A}_{k}$. In this section we develop further information about $B$ starting with the assumption that $B$ is sufficiently near $A$.

Informally, there are two types of changes which can occur as we go from $A$ to a nearby $B$. First is a small change in the conformal structure of $M(A)$ away from the points of $\operatorname{Sing}(A)$. This phenomenon will be described by a quasiconformal map of part of $M(A)$ into $M(B)$. The second type of change is a change in the structure of $A$ near the singular points. Presumably this type of deformation can be described using the algebraic theory of deformation of singularities. However, the situation is unclear except in simple cases. 
We begin by noting that if $A$ has no singular points (i.e., $\operatorname{Sing}(A)=\varnothing$ ) then the change from $A$ to a nearby $B$ is exactly that induced by a small change in the conformal structure of $M(A) \backslash \partial A$.

Recall that for $S, S^{\prime}$ in $\mathscr{S}$, the Teichmüller distance between $S$ and $S^{\prime}$ is defined by

$$
\begin{array}{r}
d_{\tau}\left(S, S^{\prime}\right)=\inf \{\log K ; \text { there is a } K \text {-quasiconformal } \\
\text { homeomorphism of } \left.S \text { and } S^{\prime}\right\} .
\end{array}
$$

TheOrem 4.1. Suppose $S$ is in $\mathscr{S}$ and $A=A(S)$. There is an $\varepsilon=\varepsilon(A)$ $>0$ such that the set of function algebras $B$ with $d(A, B)<\varepsilon$ consists entirely of algebras of the form $B=A\left(S^{\prime}\right)$ for some $S^{\prime}$ in $\mathscr{S}$. Furthermore, for such $B, d(A, B) \sim d_{\tau}\left(S, S^{\prime}\right)$. Also, if $S^{\prime \prime}$ is any element of $\mathscr{S}$ with $d_{\tau}\left(S, S^{\prime \prime}\right)$ sufficiently small then $d\left(A, A\left(S^{\prime \prime}\right)\right)<\varepsilon$.

Proof. First select $\varepsilon$ so small that, by Corollary 3.5, we know $B$ is in $\mathscr{A}_{k}$. By Proposition 2.5 we know that for any such $B$

$$
k(A)=k(B) \geq \operatorname{dim} H^{1}(M(B), \mathbf{R}) .
$$

By Corollary 7.7 of [15],

$$
\operatorname{dim} H^{1}(M(), \mathbf{R}) \text { is lower semi-continuous on } \mathscr{A}_{k} .
$$

Hence $k(B)=\operatorname{dim} H^{1}(M(B), \mathbf{R})$ for all $B$ in an open neighborhood of $A$. As noted in Proposition 2.5, this insures that $B=A\left(S^{\prime}\right)$ for some $S^{\prime}$ in $\mathscr{S}$. The result now follows from the fact that for algebras of the form $A\left(S^{\prime}\right)$, $S^{\prime}$ in $\mathscr{S}$, the Banach-Mazur metric is equivalent, in the small, to the Teichmüller distance [12].

We now prove an analogous result for algebras with singularities. The basic idea is that if the constants are allowed to depend on $A$ then the basic construction of $\sigma$ in the proof of Theorem 3.1 can be carried out for points which are not close to pre-images of images of branch points of $G$.

THEOREM 4.2. Let $A$ be in $\mathscr{A}_{k}$. There is a finite set $R$ in $M(A)$ with $\operatorname{Sing}(A) \subseteq R$ and the cardinality of $R$ dominated by a function of $k$ alone such that given any open neighborhood $N$ of $R$ there are constants $\varepsilon_{0}$ and $c$ (both of which depend on $A$ and $N$ ) so that the following holds. If $B$ is a uniform algebra with $d(A, B)<\varepsilon<\varepsilon_{0}$ then there is a $T$ in $L(A, B)$, an 
open set $U$ in $M(B), \partial B \subseteq U$, and a map $\tau$ of $U$ into $M(A)$ so that

(4.1) $T 1=1$

(4.2) $\|T f g-T f T g\| \leq c \varepsilon\|f\|\|g\|$ for all $f, g$ in $A$.

(4.3) $|T f(x)-f(\tau(x))| \leq c \varepsilon\|f\|$ for all $f$ in $A, x$ in $U$.

(4.4) $\tau$ is a continuous map of $U$ onto $M(A) \backslash N$.

(4.5) on $U \backslash \partial B, \tau$ is a quasiconformal homeomorphism and $\sup _{U \backslash \partial B} \mid$ dilatation of $\tau \mid \leq 1+c \varepsilon$.

Note. The proof actually shows that if $T$ is given so that (4.1) and (4.2) hold, then $\tau$ can be constructed so that (4.3), (4.4) and (4.5) are satisfied (with a different constant $c$ ).

Proof. We start with $\varepsilon_{0}$ so small that Corollary 3.5 applies and the results of [15] apply. Further conditions on $\varepsilon_{0}$ will be implicit in the proof. Using Corollary 3.5 and Theorem 3.1, we may assume that $B$ is in $\mathscr{A}_{k}$ and that we have a $T$ in $L(A, B)$ which satisfies (4.1) and (4.2) and a homeomorphism $\tau$ of $\partial B$ to $\partial A$ so that (4.3) is satisfied for $x$ in $\partial B$.

Pick and fix a function $G$ in $A$ which satisfies the conclusions of Proposition 2.5. Thus $G$ maps $M(A)$ onto $D$ in a $J$ to one manner (when multiplicity is taken into account) and $G$ vanishes on $\operatorname{Sing}(a)$ (and possibly at other points). Let $R$ be the preimages of points over which $G$ is not strictly $J$ to $1 ; R=\left\{p \in M(A) ; \operatorname{card}\left\{G^{-1}(G(p))\right\}<J\right\}$. Note that $\operatorname{Sing}(A) \subseteq R$. The integer $J$ and all the topological data of $M(A)$ can be estimated using only the integer $k$. Hence, using the Riemann-Hurwitz formula (e.g., (2) of [12]), the cardinality of $R$ can be estimated using only $k$.

Pick $\delta$ so small that for each $p$ in $R$, the set

$$
\left\{q \in M(A) ;\left|(G(q)-G(p))(1-\overline{G(p)} G(q))^{-1}\right|<\delta\right\}
$$

splits into connected components, each of which contains exactly one point of $R$. Denote the component of this set which contains $p$ by $M_{p}$. By choosing $\delta$ to be smaller still, we can insure that $M_{p} \subseteq N$. Finally, by choosing $\delta$ to be smaller still, we can insure that the sets $G\left(M_{p}\right), p$ in $R$, are disjoint or identical. Let $D_{0}=D \backslash\left\{\partial D \cup\left(\cup_{p \in R} G\left(M_{p}\right)\right)\right\}$. Let $S_{0}=$ $G^{-1}\left(D_{0}\right)$.

LEMMA 4.3. There is a constant $M$ so that if $z$ is in $D_{0},\left\{G^{-1}(z)\right\}=$ $\left\{p_{1}, \ldots, p_{J}\right\}$, then there are functions $H_{1}, \ldots, H_{J}$ in $A$ such that

$$
\begin{gathered}
H_{i}\left(p_{J}\right)=\delta_{i,}, \quad 1 \leq i, j \leq J, \\
\left\|H_{i}\right\| \leq M, \quad i=1, \ldots, J .
\end{gathered}
$$


Proof of the Lemma. The construction at the beginning of the proof of Theorem 3.1 shows how to construct the required $H_{t}$ for all $z$ near $\partial D$ and with $M=2$. Pick $z$ outside of the neighborhood $N_{0}$ of $\partial D$ on which that construction works. Let $\left\{G^{-1}(z)\right\}=\left\{p_{1}, \ldots, p_{J}\right\}$. Given $i, j, i \neq j$, we can find, by Proposition 2.5, functions $F_{i j}$ in $A$ so that $F_{i j}\left(p_{i}\right)=0$ and $F_{i j}\left(p_{j}\right) \neq 0$. By taking appropriate scalar multiples of products of the $F_{i j}$ we can obtain functions $H_{i}^{z}$ which satisfy (4.6) and satisfy (4.7) with a constant $M_{z}$ which depends on $z$. Now note that there is a neighborhood $N_{z}$ of $z$ such that for each $w$ in $N_{z}$ we can find $H_{i}^{w}$ which satisfy (4.6) and (4.7) on the set $\left\{G^{-1}(w)\right\}$ with the constant $M=2 M_{z}$. To see this, denote by $g_{i}, i=1, \ldots, J$, the $J$ analytic choices of $G^{-1}$ near $z$, normalized by $g_{l}(z)=p_{l}$. The required $H_{i}^{w}$ are obtained by applying the matrix $\left(\left(H_{i}^{z} g_{j}\right)(w)\right)^{-1}$ to the vector $\left(H_{i}^{z}, \ldots, H_{J}^{z}\right)$. The existence of the required neighborhood is insured by continuity.

The neighborhood $N_{0}$ of $\partial D$ together with these $N_{z}$ give a cover of $\bar{D}_{0}$. We extract a finite subcover and select for $M$ of (4.7) the maximum of the associated estimates. This completes the proof of the lemma.

Let $p$ be any point of $S_{0}$. Let $G_{p}=(G-G(p))(1-\overline{G(0)} G)^{-1}$. Let $H_{\imath}$ be the functions associated with the point $G(p)$ by the previous lemma. Thus, if $f$ is in $A$, then

$$
f=\sum a_{i} H_{i}+G_{p} r
$$

with scalars $a_{\imath}\left(a_{i}=f\left(p_{i}\right)\right)$ and $r$ in $A$. ( $r$ is in $A$ by Lemma 2.4.) Using (4.7), (4.8) and the fact that $|G|=1$ on $\partial A$ we obtain

$$
\left|a_{i}\right| \leq\|f\|, \quad\|r\| \leq M(J+1)\|f\| .
$$

With the decomposition (4.8) and estimates (4.9) as substitutes for the decomposition (3.6) and estimates (3.7), the construction of $\sigma\left(=\tau^{-1}\right)$ as in the proof of Theorem 3.1 goes through unchanged. The same argument as those used in that proof shows that $\tau$ has the required mapping properties (4.3) and (4.4).

The calculation which shows that $\tau$ is quasi-conformal is done in Lemma 3.4 of [16].

\section{The proof is complete.}

It seems to be a flaw that the previous proof allows the possibility that $R$ is strictly larger than $\operatorname{Sing}(A)$. Although we do not know how to improve the previous result to $R=\operatorname{Sing}(A)$, there are various ways in which the result can be improved. For instance, the map $\tau$ can be extended across the parts of $N$ which do not contain points of $\operatorname{Sing}(A)$ so 
that (4.5) still holds (but not necessarily (4.3)). Here is a sketch of one way to accomplish this. Suppose $p_{0}$ is in $R \backslash \operatorname{Sing}(A)$. By Proposition 2.3, we can find an $F$ in $A$ so that $F\left(p_{0}\right)=0$ and $F$ has no other zeros. Without loss of generality $F^{-1}(D \backslash \partial D)$ is a neighborhood of $p_{0}$ on which $F$ is one-to-one. Suppose $T$ is an element of $L(A, B)$ which satisfies (4.1) and (4.2). If $\varepsilon$ is small then the map $\lambda$ of points $p$ near $p_{0}$ into $M(B)$ given by

$$
\lambda(p)=(T F)^{-1}(F(p))=(T(F-F(p)))^{-1}(0)
$$

is a well-defined map of $N_{0}=F^{-1}(\{z ;|z|<1 / 2\})$ into $M(B)$ which satisfies for all $H$ in $A$, all $p$ in $N_{0}$

$$
|T H(\lambda(p))-H(p)| \leq c \varepsilon\|H\| .
$$

The fact that $\lambda$ is well defined and satisfies (4.10) follows from a simplified version of the proof of Theorem 3.1 or, alternatively, Proposition 8.1 of [15]. $\lambda$ is explicitly exhibited as a composite of analytic functions and thus is analytic. The composite $\tau^{-1} \circ \lambda$ (where $\tau$ is given in the previous theorem) is a quasiconformal map of a ring about $p_{0}$ to another such ring. Using extension theorems for quasiconformal maps (page 86 of [7]) it is then possible to extend $\tau$, across $N_{0}$ so that (4.4) and (4.5) remain true. It is not clear how to adapt this approach to obtain (4.4), (4.5) and (4.3). (The theory of quasiconformal maps seems to show that $\tau$ produces this way will satisfy

$$
|T f(x)-f(\tau(x))| \leq c \varepsilon^{\alpha}\|f\|
$$

for some positive constant $\alpha$.)

We will regard $\tau$ in Theorem 4.2 as extended across all such sets $N_{0}$ so that (4.4) and (4.5) hold.

Associated with the change from $A$ to $B$ are changes associated with the structure of $M(A)$ near the points in $\operatorname{Sing}(A)$ and/or changes in the behavior of the functions in $A$ near those points. The previous theorem and discussion indicate that the analysis of the changes near points in Sing $(A)$ can be localized to neighborhoods of the individual points. The next three propositions describe explicitly the changes that can happen to the three simplest singularities. The explicit constructions in [14] and in the verification of the properties of Example 7.9 is [15] show that all of the geometric possibilities allowed by these propositions can actually occur.

We start with the simplest singularity. Suppose $A$ is in $\mathscr{A}_{k}$ and $p$ in $\operatorname{Sing}(A)$ is a node, the removal of which would disconnect $M(A)$. Thus there are two connected, possibly singular, finite bordered Riemann surfaces $S_{1}$ and $S_{2}$, and points $p_{i}$ in $S_{i} \backslash \partial S_{i}, i=1,2$, so that $M(A)$ is $S_{1} \cup S_{2}$ with the points $p_{1}$ and $p_{2}$ identified to the single point $p$. Let $A_{i}$ be 
the algebra $A$ restricted to $S_{i}$. For $i=1,2, A_{i}$ is in $\mathscr{A}$ and $p_{i}$ is not in $\operatorname{Sing}\left(A_{i}\right)$. Let $A_{1} \oplus A_{2}$ be the algebra pairs $\left(f_{1}, f_{2}\right)$ with $f_{i}$ in $A_{i}$. We will regard $f_{i}$ as defined on $S_{1} \cup S_{2}$ by setting $f_{l}=0$ on $S_{j}, j \neq i$. Hence $A$ is the subalgebra of $A_{1} \oplus A_{2}$ of pairs $\left(f_{1}, f_{2}\right)$ which satisfy $f_{1}\left(p_{1}\right)=f_{2}\left(p_{2}\right)$. By Proposition 2.3, we can find $f_{i}$ in $A_{i}$ which vanish at $p_{i}$ and at no other point of $S_{i}$. Select such $f_{i}$ and let $X_{1}=\left(f_{1}, 0\right), X_{2}=\left(0, f_{2}\right)$. We have

$$
X_{1}, X_{2} \in A, \quad X_{1}(p)=X_{2}(p) \text {. }
$$

Also, for all $g$ in $A$ there are $g_{1}, g_{2}$ in $A$ so that

$$
g=g(p)+X_{1} g_{1}+X_{2} g_{2} \text {. }
$$

If we further require that $X_{2} g_{1} \equiv X_{1} g_{2} \equiv 0$ then the $g_{1}$ are uniquely determined and depend linearly and continuously on $g$, i.e., for all $g$ in $A$

$$
\left\|g_{\imath}\right\| \leq c\|g\|, \quad i=1,2 .
$$

The constant $c$ depends only on the choices of $X_{1}$ and $X_{2}$. Finally

$$
X_{1} X_{2} \equiv 0 \text {. }
$$

The situation summarized by (4.11)-(4.14) completely characterizes this type of singularity.

For sufficiently small positive $\delta,\left\{p \in M(A) ;\left|X_{1}(p)+X_{2}(p)\right|=\delta\right\}$ is a union of two curves $\gamma_{1}, \gamma_{2}$ with $\gamma_{i} \subseteq S_{i}, \gamma_{i}=\left\{p \in M(A) ;\left|X_{i}\right|=\delta\right.$, $\left|X_{j}\right|=0, j \neq i$ and $\gamma_{i}$ bounds a small neighborhood of $p_{i}$ in $S_{i} \cdot \gamma=\gamma_{1} \cup \gamma_{2}$ bounds a small neighborhood $M$ of $p$. Topologically $M$ is two disks with their centers identified. Choose $\delta$ so small that $M$ contains no points of the set $R$ of Theorem 4.2 other than $p$. We now apply Theorem 4.2 to $A$. We select the set $N$ so that $N \cap M \subseteq\left\{p ;\left|X_{1}+X_{2}\right| \leq \delta / 10\right\}$. Suppose now that $B$ is a uniform algebra close to $A$. We wish to describe the points of $M(B)$ which "correspond" to the points of $M$ in $M(A)$.

Proposition 4.4. There are constants $\varepsilon_{1}, c_{1}, c_{2}$ (which depend on $A, X_{1}$, $X_{2}, M$, and $N$ ) so that if $B$ satisfies the hypotheses of Theorem 4.2, and $d(A, B)<\varepsilon<\varepsilon_{1}$ then the following additional conclusions can be drawn.

There are $\tilde{X}_{1}$ and $\tilde{X}_{2}$ in $B$ such that

$$
\left\|T X_{i}-\tilde{X}_{i}\right\| \leq c_{1} \varepsilon, \quad i=1,2 .
$$

There are linear maps $R_{i}$ of $B$ to itself, $i=1,2$, and a linear functional $L$ on $B$ so that for all $f$ in $B$

$$
f=L(f)+\tilde{X}_{1} R_{1}(f)+\tilde{X}_{2} R_{2}(f) .
$$


These operators satisfy $X_{2} T^{-1} R_{1}(f) \equiv 0$ and $X_{1} T^{-1} R_{2}(f) \equiv 0$ for all $f$, and are bounded

$$
\|L\| \leq c_{1},\|R,\| \leq c_{1}, \quad i=1,2 .
$$

Furthermore there is a constant $\alpha,|\alpha|<c_{1} \varepsilon$ so that

$$
\tilde{X}_{1} \tilde{X}_{2} \equiv \alpha .
$$

Finally, the map of $M(B)$ into $\mathbf{C}^{2}$ which sends $p$ to $H(p)=\left(p\left(\tilde{X}_{1}\right), p\left(\tilde{X}_{2}\right)\right)$ is a one-to-one analytic map of

$$
\left\{p \in M(B) ;\left|\tilde{X}_{\imath}(p)\right|<c_{2} \text { for } i=1,2\right\}
$$

onto

$$
E=\left\{\left(z_{1}, z_{2}\right) \in C^{2} ;\left|z_{i}\right|<c_{2} \text { for } i=1,2 \text { and } z_{1} z_{2}=\alpha\right\}
$$

Proof. First we construct $\tilde{X}_{t}$ which satisfy (4.15) and (4.18). We do this by an iterative process which will converge if $\varepsilon$ is sufficiently small.

For $i=1,2$, let $L_{\imath}$ be the linear map of $A$ to $A$ which sends $g$ in $A$ to the function $g_{1}$ of (4.12).

The construction is most easily described in terms of the multiplication on $B$ pulled back to $A$ by $T$. That is, let $\times$ be defined by

$$
f \times g=T^{-1}(T f T g) .
$$

Since $X_{1} X_{2} \equiv 0$, by (4.2)

$$
X_{1} \times X_{2}=r
$$

for some $r$ with $\|r\| \leq c \varepsilon$. By (4.12)

$$
X_{1} \times X_{2}=r(p)+X_{1} L_{1}(r)+X_{2} L_{2}(r) .
$$

Using the estimates on $r,(4.13)$ and (4.2), this gives

$$
X_{1} \times X_{2}=r(p)+X_{1} \times L_{1}(r)+X_{2} \times L_{2}(r)+r^{\prime},
$$

with $\left\|r^{\prime}\right\| \leq c \varepsilon^{2}$. Thus

$$
\left(X_{1}-L_{2}(r)\right) \times\left(X_{2}-L_{1}(r)\right)=r(p)+r_{1}
$$

for some $r_{1}$ with $\left\|r_{1}\right\| \leq c \varepsilon^{2}$. Set $X_{1}^{(1)}=X_{1}-L_{2}(r)$ and $X_{2}^{(1)}=X_{2}-L_{1}(r)$. Then

$$
X_{1}^{(1)} \times X_{2}^{(1)}=r(p)+r_{1} .
$$

We now iterate this construction. The next step is $X_{1}^{(2)}=X_{1}^{(1)}-L_{2}\left(r_{1}\right)$, $X_{2}^{(2)}=X_{2}^{(1)}-L_{1}\left(r_{1}\right)$. Then

$$
X_{1}^{(2)} \times X_{2}^{(2)}=r(p)+r_{1}(p)+r_{2}
$$


and $\left\|r_{2}\right\| \leq c \varepsilon^{3}$. Denoting the limit, $\lim _{n} X_{i}^{(n)}$ by $X_{t}^{\infty}, i=1,2$, we obtain

$$
X_{1}^{\infty} \times X_{2}^{\infty}=\alpha
$$

for some constant $\alpha,|\alpha|<c \varepsilon$. If we set $\tilde{X}_{l}=T X_{l}^{\infty}$ for $i=1,2$ then (4.15) and (4.18) are satisfied. By (4.12), (4.13), (4.15) and (4.2), if $f$ is in $A$ then

$$
f=f(p)+X_{1}^{\infty} \times L_{1}(f)+X_{2}^{\infty} \times L_{2}(f)+r
$$

where the "error term" $r$ satisfies $\|r\| \leq c \varepsilon$. We apply the decompositon (4.21) to $r$ and add the resulting quantity to (4.21). This yields

$$
f=f(p)+r(p)+X_{1}^{\infty} \times L_{1}(f+r)+X_{2}^{\infty} \times L_{2}(f+r)+r^{\prime}
$$

with $\left\|r^{\prime}\right\| \leq c \varepsilon^{2}$. Iteration of this process and passage to the limit (since the process converges for small $\varepsilon$ ) yields

$$
f=c(f)+X_{1}^{\infty} \times L_{1}\left(S_{1}(f)\right)+X_{2}^{\infty} \times L_{2}\left(S_{2}(f)\right)
$$

where $c$ is a bounded linear functional and the $S_{i}$ are linear operators with $\left\|I-S_{i}\right\| \leq c \varepsilon$. If we apply $T$ to this equation and take note of (4.19) we obtain an equation which can be rewritten in the form (4.16) and satisfies (4.17).

Since we have assumed $\varepsilon$ is small, we know by the results of the previous section that $B$ is in $\mathscr{A}$. Hence the functions in $B$ are analytic on $M(B) \backslash \partial B$. Thus the map $H(p)$ is an analytic map which, by (4.18) goes into the required set. Now note that if $p$ is in $M(B)$ and $\left|\tilde{X}_{i}(p)\right|$ is small for $i=1,2$ then, by (4.16) and (4.17), the value of $f(p)$ is completely determined by these values. That is, $n$-fold iteration of the decomposition produces

$$
f=Q+\sum_{k=0}^{n} \tilde{X}_{1}^{n-k} \tilde{X}_{2}^{k} S_{k}(f)
$$

where $Q$ is a polynomial in $\tilde{X}_{1}$ and $\tilde{X}_{2}$ and the $S_{k}$ are linear maps with

$$
\sum_{k=0}^{n}\left\|S_{k}\right\| \leq c^{n}
$$

for some constant $c$ which depends only on the constant $c_{1}$ in (4.17). Hence, if $\left|\tilde{X}_{i}(p)\right| \leq \frac{1}{2} c$ then the value of $f(p)$ is determined by the numbers $\tilde{X}_{l}(p)$. Thus $H$ is one-to-one on the indicated domain.

We now show that if $c_{2}$ is small then the image of the map $H$ is all of the set $E$. First, for convenience, suppose that $\alpha$ in (4.18) is not zero. Let $\tau$ be the map produced in Theorem 4.2. For $i=1,2, \tau^{-1}\left(\gamma_{i}\right)$ are curves in $M(B)$ and for points $x$ on $\gamma_{i}$,

$$
\left|T\left(x_{i}^{\infty}\right)\left(\tau^{-1}(x)\right)-X_{i}^{\infty}(x)\right| \leq c \varepsilon\left\|X_{i}^{\infty}\right\|
$$


If we now use the fact that $T X_{i}^{\infty}=\tilde{X}_{i}$ and use (4.15), we obtain

$$
\left|\tilde{X}_{i}\left(\tau^{-1}(x)\right)-X_{i}(x)\right| \leq c \varepsilon\left\|X_{i}\right\| .
$$

Hence, for $x$ on $\gamma_{i}$,

$$
\left|\tilde{X}_{t}\left(\tau^{-1}(x)\right)\right|=\delta+O(\varepsilon) .
$$

( $\delta$ is the parameter used to define $\gamma_{l}$.) Hence, if $\delta$ was selected to be small, then this together with (4.18) insures that for $x$ in $\gamma_{1} \cup \gamma_{2}, H(x)$ is in $E$. By assumption, at each point of $\partial A$, either $\left|X_{i}\right| \geq 1$ or $\left|X_{2}\right| \geq 1$. Hence, if $\varepsilon$ is small and $c_{2}<1 / 2$, then the points of $\partial A$ are not mapped into $E$ by $H$. We have seen that $H(M(A)) \cap E$ is not empty. Since the map $H$ is open or constant on each of the Riemann surfaces which make up $M(A) \backslash \partial A$, and since $H(\partial A) \cap E=\varnothing, H(M(A)) \cap E$ must be open. Since $H$ is continuous and $M(A)$ is compact, $H(M(A)) \cap E$ is relatively closed. Thus, since $E$ is connected, we conclude that $E \subseteq H(M(A))$.

Finally, if $\alpha=0$, we argue as we just did to show separately that both $E_{1}=\left\{\left(z_{1}, 0\right) ;\left|z_{2}\right|<c_{2}\right\}$ and $E_{2}=\left\{\left(0, z_{2}\right) ;\left|z_{2}\right|<c_{2}\right\}$ are in $H(M(A))$.

The proof is complete.

The main thrust of this proposition is that near $p, M(A)$ contains part of the singular algebraic curve $z_{1} z_{2}=0$ and that the corresponding region is the spectrum of the "deformed algebra" $B$ is part of the "deformed curve" $z_{1} z_{2}=\alpha$ (which is generally non-singular). Another way to describe the geometry of this situation is in terms of covering maps of the disk. On $M(A)$ we consider two functions $h=X_{1}+X_{2}$ and $k=X_{1}-X_{2}$. These functions separate points near $p$ and satisfy the equation $h^{2}=k^{2}$. Thus the function $h$ exhibits the region of $M(A)$ near $p$ as a two sheeted covering of a region near the origin in the $k$ plane and the singularity of this covering is a double point over the origin. The analogous functions in $B$ are $\tilde{h}=\tilde{X}_{1}+\tilde{X}_{2}$ and $\tilde{k}=\tilde{X}_{1}-\tilde{X}_{2}$. They satisfy $\tilde{h}^{2}=\tilde{k}^{2}+4 \alpha$. Thus $\tilde{h}$ exhibits a region on $M(B)$ as a two sheeted covering of a region near the origin of the $\tilde{k}$ plane. This covering map has simple branch points over $\pm 2 \alpha$. (A double covering of the disk branched over $\pm 2 \alpha$ is seen to be conformally equivalent to a ring $\{w ; \beta<|w|<1\}$ where $\beta$ is a parameter which is determined by $\alpha$ and tends to zero as $\alpha$ tends to zero.) Thus the double point in $M(A)$ has been replaced by a thin neck.

On $M(A)$, removal of the point $p$ or the neighborhood of $p$ bounded by $\gamma_{1} \cup \gamma_{2}$ would disconnect $M(A) . M(B)$ has a similar property; removal of the region bounded by the curves $\tau^{-1}\left(\gamma_{1}\right) \cup \tau^{-1}\left(\gamma_{2}\right)$ (i.e., the region where both $\tilde{X}_{\imath}$ are small) disconnects $M(B)$. To see this, for $i=1,2$, let

$$
U_{i}=\left\{p \in M(B) ;\left|\tilde{X}_{l}(p)\right|>\frac{1}{2} \min \left(c_{2}, \frac{1}{2}\right)\right\}
$$


and let

$$
V=\left\{p \in M(B) ;\left|\tilde{X}_{i}(p)\right| \leq \frac{1}{2} c_{2} \text { for } i=1 \text { and } i=2\right\} .
$$

The $U_{t}$ are open and non-empty. By (4.18) they are disjoint. Since $\left(M(B) \backslash U_{1}\right) \cap\left(M(B) \backslash U_{2}\right) \subseteq V$, the removal of $V$ disconnects $M(B)$.

Finally we should note the interpretation that goes with the case $\alpha=0$ of (4.18). In that case $B$ has a realization as a subalgebra of codimension one of $B_{1} \oplus B_{2}$ for $B_{l}$ in $\mathscr{A}$. There is a singularity in $M(B)$ of the same sort as that at $p$ in $M(A)$. Furthermore $d\left(A_{i}, B_{i}\right) \leq c \varepsilon$ for $i=1,2$. In this case the passage from $A$ to $B$ consists of deforming both $A_{1}$ and $A_{2}$ and a slight change in the selection of the points $p_{i}$.

We now do a similar analysis for the cusp singularity. This type of singularity is obtained by taking the kernel of a derivation at a non-singular point. We start with $A^{+}$in $\mathscr{A}$ and $p_{0}$ in $M\left(A^{+}\right) \backslash\left(\partial A^{+} \cup \operatorname{Sing}\left(A^{+}\right)\right)$. By Proposition 2.3 we can find $f_{0}$ in $A^{+}$which has a simple zero at $p_{0}$ and no other zeros. We assume $\left|f_{0}\right| \geq 1$ on $\partial A^{+}$. Since $f_{0}$ gives a local coordinate at $p_{0}$, we may use it to describe the point derivations at $p_{0}$. Any $g$ in $A^{+}$ can be written near $p_{0}$ as $g=h \circ f_{0}$ for some function $h$ analytic near zero. Any point derivation at $p$ is a scalar multiple of the functional $D$ defined by $D g=h^{\prime}(0)$ (i.e., $D g=\left(d g / d f_{0}\right)\left(p_{0}\right)$.) Let $A$ be the subalgebra of $A^{+}$ consisting of those $g$ in $A^{+}$with $D g=0$. Equivalently, (using Lemma 2.4)

$$
A=\left\{g \in A^{+} ; g=c+f_{0}^{2} h, c \in \mathbf{C}, h \in A^{+}\right\} .
$$

Suppose we start with $g$ in $A$ and write $g=c+f_{0}^{2} h$ with $c$ in $\mathbf{C}$ and $h$ in $A^{+}$. The function $h$ can be written as $c_{1}+c_{2} f_{0}+f_{0}^{2} k$ for scalars $c_{l}$ and some $k$ in $A^{+}$. Combining these representations we find that any $g$ in $A$ can be written as

$$
g=c+c_{2} f_{0}^{3}+f_{0}^{2} h
$$

with $h$ in $A$. Thus $f_{0}^{2}$ and $f_{0}^{3}$ generate the ideal of functions in $A$ which vanish at $p_{0}$. We now set $W=f_{0}^{2}, V=f_{0}^{3}$. The previous equation can be rewritten as

$$
g=r_{1}(g)+r_{2}(g) V+W R(g)
$$

with $r_{1}$ and $r_{2}$ linear functionals, $R$ a linear map of $A$ to itself, and all three bounded:

$$
\left\|r_{i}\right\| \leq c, \quad i=1,2 \text { and }\|R\| \leq c .
$$

(4.22) and (4.23) are the analogs of (4.12) and (4.13). The equation relating the generators of the ideal is more complicated than (4.14). $V$ and $W$ satisfy

$$
V^{2}=W^{3}
$$


The main content of the next proposition is that a small deformation of $A$ changes this singular point to a subset of a (possibly degenerate) elliptic curve given by an equation

$$
\tilde{V}^{2}=\tilde{W}^{3}+a \tilde{W}+b
$$

for small scalars $a$ and $b$.

We wish to use Theorem 4.2 on $A$. Select $\delta$ to be sufficiently small so that $\gamma=\left\{p ;\left|f_{0}(p)\right|=\delta\right\}$ is a simple closed curve in $M(A)$ which surrounds a neighborhood $M$ of $p_{0}$ in $M(A)$ and $M$ contains no other points of the set $R$ of Theorem 4.2. Select $N$ so that $N \cap M \subseteq\left\{\left|f_{0}\right|<\right.$ $\delta / 10\}$. We now suppose $B$ is a uniform algebra with $d(A, B)$ sufficiently small so that we can use Theorem 4.2. Thus $d(A, B)<\varepsilon<\varepsilon_{0}$ and we obtain $T$ and $\tau$ which satisfy (4.1)-(4.5). We now wish to describe the points in $M(B)$ which correspond to $M$ in $M(A)$.

Proposition 4.5. There are constants $\varepsilon_{1}, c_{1}$ and $c_{2}$ (which depend on $A$, $f_{0}, M$, and $\left.N\right)$ so that if $B$ satisfies the hypotheses of Theorem 4.2 and $T$ and $\tau$ satisfy the conclusions of Theorem 4.2 and $d(A, B)<\varepsilon<\varepsilon_{1}$ then the following additional conclusions can be drawn.

There are $\tilde{V}$ and $\tilde{W}$ in $B$ with

$$
\|T V-\tilde{V}\|<c_{1} \varepsilon, \quad\|T W-\tilde{W}\|<c_{1} \varepsilon .
$$

There are linear functionals $\tilde{r}_{t}, i=1,2$, and a linear map $\tilde{R}$ of $B$ to itself so that for all $f$ in $B$

$$
f=\tilde{r}_{1}(f)+\tilde{r}_{2}(f) \tilde{V}+\tilde{W} \tilde{R}(f) .
$$

These maps are bounded

$$
\left\|\tilde{r}_{\imath}\right\| \leq c_{1}, \quad i=1,2 ; \quad\|\tilde{R}\| \leq c_{1} .
$$

There are constants $\alpha_{1}, \alpha_{2}$ which satisfy $\left|\alpha_{i}\right| \leq c_{1} \varepsilon, i=1,2$, so that

$$
\tilde{V}^{2}=\tilde{W}^{3}+\alpha_{1} \tilde{W}+\alpha_{2} .
$$

Finally, the map $H$ of $M(B)$ into $\mathbf{C}^{2}$ given by $H(p)=(\tilde{V}(p), \tilde{W}(p))$ is a one-to-one analytic map of

$$
M(B) \cap\left\{|\tilde{V}|<c_{2}\right\} \cap\left\{|\tilde{W}|<c_{2}\right\}
$$

onto the set of points $\left(z_{1}, z_{2}\right)$ in $\mathbf{C}^{2}$ which are in the set

$$
\left\{z_{1}^{2}=z_{2}^{3}+\alpha_{1} z_{2}+\alpha_{2}\right\} \cap\left\{\left|z_{1}\right|<c_{2}\right\} \cap\left\{\left|z_{2}\right|<c_{2}\right\} .
$$

Proof. The proof is in the same pattern and spirit as the proof of the previous proposition, however the computational details are more elaborate. We again use the notation $f \times g=T^{-1}(T f T g)$ for $f, g$ in $A$. We 
will write $O\left(\varepsilon^{n}\right)$ to represent any function in $A$ or $B$ which satisfies a norm estimate $\left\|O\left(\varepsilon^{n}\right)\right\| \leq c \varepsilon^{n}$ where $\varepsilon$ is the small number in the hypotheses of the proposition and $c$ is a universal constant (which changes from use to use).

Lemma 4.6. Suppose $V_{1}$ and $W_{1}$ are in $A$ and satisfy $\left\|V-V_{1}\right\| \leq \varepsilon$, $\left\|W-W_{1}\right\| \leq \varepsilon$. Then there ae linear functionals $k_{i}$ on $A, i=1, \ldots, 6$, and $a$ linear map $S$ of $A$ to $A$ such that any $f$ in $A$ can be written as

$$
\begin{aligned}
f= & k_{1}(f)+k_{2}(f) V_{1}+k_{3}(f) W_{1}+k_{4}(f) V_{1} W_{1} \\
& +k_{5}(f) W_{1}^{2}+k_{6}(f) V_{1} W_{1}^{2}+W_{1}^{3} S(f) .
\end{aligned}
$$

Furthermore these operators are bounded

$$
\begin{aligned}
\left\|k_{i}\right\| & \leq c, \quad i=1, \ldots, 6, \\
\|S\| & \leq c
\end{aligned}
$$

and the bounds can be chosen so as not to depend on $\varepsilon$ (if $\varepsilon$ is small) but only on the norm estimates in (4.23).

Proof of the Lemma. Three applications of the decomposition (4.23); first to $f$, then to $R(f)$, then to $R(R(f))$ produce the desired result for the case $V=V_{1}, W=W_{1}$. We now perturb that result from $(V, W)$ to $\left(V_{1}, W_{1}\right)$. Start with $f$ written in the form (4.29) with $V$ and $W$ in the places of $V_{1}$ and $W_{1}$. Replace $V$ with $V_{1}+O(\varepsilon)$ and $W$ with $W_{1}+O(\varepsilon)$. Multiplication and collecting terms from the resulting expression produces an equation of the form

$$
f=g+O(\varepsilon)
$$

with $g$ of the required form (involving $V_{1}$ and $W_{1}$ ). We now apply the decomposition (4.30) to the error term $O(\varepsilon)$. When this is combined with (4.30) we obtain

$$
f=g_{2}+O\left(\varepsilon^{2}\right)
$$

where $g_{2}$ is of the required form. Iteration of this process produces a sequence of $g_{n}$ which converge to the required decomposition. This proves the lemma.

We now apply the decomposition (4.29) to the function $f=W \times W$ $\times W-V \times V$. By (4.2) and (4.24) we know that $f=O(\varepsilon)$. Hence

$$
\begin{aligned}
W \times W \times W-V \times V= & c_{1}+c_{2} V+c_{3} W+c_{4} V W \\
& +c_{5} W^{2}+c_{6} V W^{2}+W^{3} S(f)
\end{aligned}
$$


for small constants $c_{i},\left|c_{i}\right| \leq c \varepsilon, i=1, \ldots, 6$. We now make two types of changes on the right hand side of (4.31). First we change the type of multiplication; that is, we replace $V W$ by $V \times W, W^{2}$ by $W \times W, V W^{2}$ by $V \times W \times W$ and $W^{3} S(f)$ by $W \times W \times W \times S(f)$. Using (4.2) and the estimates on the $c_{i}$ and $S(f)$ we see that this produces an error term of the form $O\left(\varepsilon^{2}\right)$. The second change is to replace $W=W_{0}$ by a new element $W_{1}$ which is related by

$$
W_{0}=W_{1} \times\left(1+\frac{1}{3} S(f)\right) .
$$

(Since $S(f)$ is small $T\left(1+\frac{1}{3} S(f)\right)$ is an invertible element of $B$ and $W_{1}=W_{0} \times T^{-1}\left(T\left(1+\frac{1}{3} S(f)\right)^{-1}\right)$.) Rewrite $W_{0}$ as $W_{1}+\frac{1}{3} W_{1} \times S(f)+$ $O\left(\varepsilon^{2}\right)$ in the equation. After multiplying out and collecting terms we obtain, for new constants $c_{i}$,

$$
\begin{aligned}
W_{1} \times W_{1} \times W_{1}-V \times V= & c_{1}+c_{2} V+c_{3} W_{1}+c_{4} V \times W_{1} \\
& +c_{5} W_{1} \times W_{1}+c_{6} V \times W_{1} \times W_{1} \\
& +O\left(\varepsilon^{2}\right),
\end{aligned}
$$

and the constants $c_{i}$ satisfy $\left|c_{i}\right| \leq c \varepsilon$. Now write $V=V_{1}-\frac{1}{2} c_{2}-\frac{1}{2} c_{4} W$ $-\frac{1}{2} c_{6} W \times W$. When we substitute this in (4.32) and collect terms we obtain

$$
W_{1} \times W_{1} \times W_{1}-V_{1} \times V_{1}=d_{1}+d_{2} W_{1}+d_{3} W_{1} \times W_{1}+O\left(\varepsilon^{2}\right)
$$

for constants $d_{i}$ which satisfy $\left|d_{i}\right| \leq c \varepsilon$.

We now iterate the process that led from (4.24) and (4.33). First use Lemma 4.6 to expand the $O\left(\varepsilon^{2}\right)$ term in (4.33) in terms of $W_{1}$ and $V_{1}$. Next, change the multiplication in this expansion of $O\left(\varepsilon^{2}\right)$ from ordinary multiplication to the multiplication $\times$. By (4.2) this produces an error of the form $O\left(\varepsilon^{3}\right)$. The right-hand side of the resulting expression will then have as term of the form $W_{1} \times W_{1} \times W_{1} \times S\left(O\left(\varepsilon^{2}\right)\right)$. By changing $W_{1}$ to $W_{2}=W_{1}+O\left(\varepsilon^{2}\right)$ this term can be cancelled leaving an error of the form $O\left(\varepsilon^{3}\right)$. The resulting analog of (4.32) will have terms involving $V_{1}, V_{1} \times W_{2}$ and $V_{1} \times W_{2} \times W_{2}$. Those terms were produced by the last application of Lemma 4.6 and have coefficients of size $O\left(\varepsilon^{2}\right)$. Hence they can be eliminated in the same way in which (4.32) was changed to (4.33). That is, after changing $V_{1}$ to appropriate $V_{2}$ of the form $V_{2}=V_{1}+O\left(\varepsilon^{2}\right)$ we obtain

$$
W_{2} \times W_{2} \times W_{2}-V_{2} \times V_{2}=d_{1}+d_{2} W_{2}+d_{2} W_{2} \times W_{2}+O\left(\varepsilon^{3}\right)
$$

where the $d_{i}$ are new constants which satisfy $\left|d_{t}\right| \leq c \varepsilon$. Iteration of the process that goes from (4.33) to (4.34) and passage to the limit produces

$$
W_{\infty} \times W_{\infty} \times W_{\infty}-V_{\infty} \times W_{\infty}=d_{1}+d_{2} W_{\infty}+d_{3} W_{\infty} \times W_{\infty}
$$


(for still different constants $d_{i}$ ). We apply $T$ to this equation, set $\hat{W}=$ $T\left(W_{\infty}\right), \tilde{V}=T\left(V_{\infty}\right)$ and obtain

$$
\hat{W}^{3}-\tilde{V}^{2}=d_{1}+d_{2} \hat{W}+d_{3} \hat{W}^{2} .
$$

When we set $\tilde{W}=\hat{W}^{2}-d_{3} / 3$ we obtain (4.28).

The rest of the proof goes as the proof of Proposition 4.4. We omit the details.

The region on $M(B)$ bounded by $\tau^{-1}(\gamma)$; that is, the region described by (4.28), is either a compact Riemann surface of genus one (i.e., a torus) from which an analytic disk has been removed or is one of the two degenerate forms of that configuration; a disk with two points identified or a disk with a distinguished singular point at which all the functions in $B$ have vanishing derivative. These facts can be verified by geometric analysis or by noting that the algebraic equation being considered is

$$
z_{1}^{2}=z_{2}^{3}+\alpha_{1} z_{2}+\alpha_{2}
$$

and that the classical parametrization of the torus is by the Weierstrass $\wp$ function and its derivative which are related by the equation

$$
\left(\wp^{\prime}\right)^{2}=4 \wp^{3}-g_{2 \wp}-g_{3}
$$

for constants $g_{2}$ and $g_{3}$.

Roughly, the region where $\left|z_{2}\right|$ is small corresponds to the complement of a neighborhood of the pole of $\wp$ on the torus. An explicit construction of this type of deformation of an algebra $A$ is given in [14].

For certain values of the constants $\alpha_{1}$ and $\alpha_{2}$ in (4.28) the torus is degenerate. In particular if $\alpha_{1}=\alpha_{2}=0$, then the algebra $B$ has a cusp singularity inside $\tau^{-1}(\gamma)$ and $B$ can be regarded as being obtained from $A$ by first deforming the larger algebra $A^{+}$to a $B^{+}$and then moving the singular point a bit. One way to understand this type of singularity is to regard the singular point as the "shadow" of a compact component of the spectrum $M(A)$ (in this case the compact component is a torus which is attached to the rest of $M(A)$ at $\left.p_{0}\right)$. Since a compact component carries no non-constant analytic functions, this component can't be "seen" by functions in $A$.

There is another choice for $\alpha_{1}, \alpha_{2}$ which gives a singular curve for (4.35). Let $F\left(z_{1}, z_{2}\right)=z_{1}^{2}-z_{2}^{3}-\alpha_{1} z_{2}-\alpha_{2}$. If $\alpha_{2}=-(4 / 27)^{1 / 2} \alpha_{1}$ then the curve $F=0$ has a singular point (i.e., $F_{z_{1}}=0$ and $F_{z_{2}}=0$ ) at $z_{2}=$ $\pm\left(-\alpha_{1} / 3\right)^{1 / 2}$ ). If $\alpha_{1}$ is not zero then this singularity is a double point (a node) and the part of $M(B)$ inside $\tau^{-1}(\gamma)$ is a small disk with two points 
identified. This can be understand as a deformation obtained by replacing the condition of a vanishing derivative with the conditions that a difference quotient (involving nearby points) vanish. (See, for instance, example 7.9 of [15].)

The third type of singularity which we discuss is a node the removal of which doesn't disconnect $M(A)$. An alternative description is that the singular point is obtained by identifying two points on the same connected, possibly singular, finite bordered Riemann surface. From the point of view of local analytic geometry, this type of singularity is the same as the first one considered - two patches of (complex) curve crossing at a point. However, in this case, we cannot choose $X_{1}$ and $X_{2}$ which give local coordinates and satisfy (4.14). (Since $M(A)$ with the singular point removed is connected, we cannot, in general, prevent functions which satisfy (4.14) from vanishing identically.) Thus, although the local analytic geometry is the same as that described in Proposition 4.4, the algebraic expression of that geometry is more awkward.

We start with an $A^{+}$in $\mathscr{A}$ and two points $p_{1}$ and $p_{2}$ in

$$
M\left(A^{+}\right) \backslash\left(\partial A^{+} \cup \operatorname{Sing}\left(A^{+}\right)\right) .
$$

Let $f_{l}$ be functions in $A^{+}$which vanish at $p_{\imath}$ and no other points of $M\left(A^{+}\right)$ (Proposition 2.3). Let $A=\left\{g\right.$ in $\left.A^{+} ; g\left(p_{1}\right)=g\left(p_{2}\right)\right\}$. Thus $M(A)$ is obtained from $M\left(A^{+}\right)$by identifying the two points $p_{1}$ and $p_{2}$ to a single point $p_{0}$. We consider the following functions in $A$

$$
W=f_{1} f_{2}, \quad X=f_{1}^{2} f_{2}, \quad Y=f_{1} f_{2}^{2} .
$$

These functions generate the ideal of functions in $A$ which vanish at $p_{0}$. If $f$ is in $A$ then

$$
f=f\left(p_{0}\right)+r(f) X+W R(f)
$$

where $r(f)$ is a linear functional, $R$ is a linear map of $A$ to $A$ and both are bounded

$$
\|r\| \leq c, \quad\|R\| \leq c .
$$

This decomposition is obtained by writing $(f-f(p)) / W=h$ for some $h$ in $A^{+}$(Lemma 2.4) and then writing $h=c_{1}+c_{2} f_{1}+W k$ for constants $c_{1}$ and $c_{2}$ and some $k$ in $A^{+}$(Lemma 2.4 again). We obtain (4.36) by setting $r(f)=c_{2}$ and $R(f)=c_{1}+W k$. In particular $Y$ can be written

$$
\text { (4.38) } Y=c X+W g
$$

for some $c$ in $\mathbf{C}, g$ in $A, g\left(p_{0}\right) \neq 0$. If we multiply (4.38) by $X$ and use the relation

$$
X Y=W^{3}
$$


we obtain the equation

$$
W^{3}=c X^{2}+W X g
$$

with $g\left(p_{0}\right) \neq 0$. This equation is the more complicated substitute for (4.14).

Using (4.36) repeatedly, one can write the function $g$ in (4.40) as a formal power series in $X$ and $W$. By (4.37), this power series actually converges in a small neighborhood of $p_{0}$. Hence, near $p_{0}$ we can regard $g$ as a function of the two "coordinate variables" $X$ and $W$. Using this interpretation of $g$ as a function of two variables, we introduce the set

$$
E_{\boldsymbol{\theta}}=\left\{\left(z_{1}, z_{2}\right) \in \mathbf{C}^{2} ;\left|z_{1}\right| \leq \boldsymbol{\theta},\left|z_{2}\right| \leq \boldsymbol{\theta}, z_{1}^{3}=c z_{2}^{2}+z_{1} z_{2} g\left(z_{1}, z_{2}\right)\right\} .
$$

It is straightforward to verify that the map of $p$ to $(W(p), X(p))$ is an analytic map of a neighborhood $M$ of $p_{0}$ in $M(A)$ into $E_{\theta}$ for some $\theta$ and if $\theta$ is chosen to be sufficiently small and $M$ is chosen appropriately then the map is one-to-one and onto $E_{\theta}$.

As before, we wish to apply Theorem 4.2. First choose $M$ so small that $M$ contains no points of $R$ other than $p_{0}$. Select $N$ so that the part of $N$ near $p_{0}$ is inside $M$. Again, suppose that $d(A, B)$ is small and that $T$ and $\tau$ satisfy (4.1)-(4.5).

Proposition 4.7. There are constants $\varepsilon_{1}, c_{1}$, and $c_{2}$ (which depend on $A$, $N, W, X, Y)$ so that if $B$ satisfies the hypotheses of Theorem 4.2 and $T$ and $\tau$ satisfy the conclusions of that theorem and $d(A, B)<\varepsilon<\varepsilon_{1}$ then the following additional conclusions can be drawn.

There are $\tilde{X}, W$, in $B$ with

$$
\|T X-\tilde{X}\| \leq c_{1} \varepsilon, \quad\|T W-\tilde{W}\| \leq c_{1} \varepsilon
$$

and linear functionals $\tilde{r}_{i}, i=1,2$, and a linear map $\tilde{R}$ of $B$ to itself so that any $f$ in $B$ can be written.

$$
f=\tilde{r}_{1}(f)+\tilde{r}_{2}(f) \tilde{X}+\tilde{W} \tilde{R}(f) .
$$

These maps are bounded

$$
\left\|\tilde{r}_{i}\right\| \leq c_{1}, \quad i=1,2 ; \quad\|R\| \leq c_{1} .
$$

Let $L=\left\{q\right.$ in $\left.M(B) ;|\tilde{X}(q)|<c_{2},|\tilde{W}(q)| \leq c_{2}\right\}$. There is a function $\tilde{g}$ in $B$ with $c_{1}^{-1} \leq|g(q)| \leq c_{1}$ for all $q$ in $L$ and a constant $c_{0}$ so that

$$
\tilde{W}^{3}=c_{0} \tilde{X}^{2}+P(\tilde{X}, \tilde{W})+\tilde{W} \tilde{X} \tilde{g}
$$

where $P$ is a polynomial

$$
P(\tilde{X}, \tilde{W})=a_{1}+a_{2} \tilde{X}+a_{3} \tilde{W}+a_{4} \tilde{W}^{2}+a_{5} \tilde{W} \tilde{X}+a_{6} \tilde{W}^{2} \tilde{X}
$$

with small coefficients

$$
\left|a_{i}\right| \leq c_{1} \varepsilon, \quad i=1, \ldots, 7 .
$$


Finally the map $H$ of $L$ into $\mathbf{C}^{2}$ given by $H(q)=(\tilde{W}(q), \tilde{X}(q))$ is (for appropriate $\left.c_{2}\right)$, a one-to-one analytic map of $L$ onto an open subset of the ( possibly singular) curve in $\mathbf{C}^{2}$ given near $(0,0)$ by

$$
\begin{aligned}
& \tilde{E}_{\theta}=\left\{\left(z_{1}, z_{2}\right) \in \mathbf{C}^{2} ;\left|z_{1}\right| \leq \boldsymbol{\theta},\left|z_{2}\right| \leq \boldsymbol{\theta}\right. \text { and } \\
& \left.\qquad z_{1}^{3}=c_{0} z_{2}^{2}+P\left(z_{1}, z_{2}\right)+z_{1} z_{2} \tilde{g}\left(z_{1}, z_{1}\right)\right\}
\end{aligned}
$$

$(\tilde{g}($,$) is defined analogously to g()$,$) . The image of L contains \tilde{E}_{\theta}$ for some small $\boldsymbol{\theta}^{\prime}$.

Proof. The proof is essentially the same as that of the previous two propositions. We still just outline the differences.

First we need a lemma which is the analog of Lemma 4.6. We describe it informally. We need to know that given $\hat{X}$ near $X, \hat{W}$ near $W$, then given any $f$ in $A, f$ can be written

$$
f=c_{1}+c_{2} \hat{X}+c_{3} \hat{W}+c_{4} \hat{W} \hat{X}+c_{5} \hat{W}^{2}+c_{6} \hat{W}^{2} \hat{X}+\hat{W}^{3} g
$$

for suitable constants $c_{\imath}$ and function $g$ in $A$.

(4.42) follows from (4.36) in the same way as the way (4.29) follows from (4.22).

Again we write $h \times g=T^{-1}(T h T g)$ for $h, g$ in $A$. Next use the decomposition (4.42) on the function

$$
h=X \times Y-W \times W \times W
$$

which, by (4.39) and (4.2), is small. This produces an equation $h=P+$ $W^{3} g$ with $P$ a polynomial with small coefficients and $g$ in $A,\|g\| \leq c \varepsilon$. Next replace $P$ and $W^{3} g$ by the corresponding expressions involving the multiplication $\times$. This introduces a correction of the sort $O\left(\varepsilon^{2}\right)$. Next write $Y$ as $Y=Y_{1} \times(1+g)$ and multiply on both sides of the equation (using the $X$ multiplication) by the multiplicative inverse (with respect to $\times)$ of $1+g$. This produces a new equation

$$
X \times Y-W \times W \times W=P+O\left(\varepsilon^{2}\right) .
$$

Iteration and passage to the limit produces

$$
X \times Y_{\infty}-W \times W \times W=P
$$

for some appropriate polynomial with respect to the multiplication $\times$. Application of the map $T$ and setting $\tilde{X}=T X, \hat{Y}=T Y_{\infty}$ and $\tilde{W}=T W$ gives

$$
\tilde{X} \hat{Y}=\tilde{W}^{3}+\tilde{P}
$$

(4.41) now follows from (4.36) by the same argument that gave (4.16) from (4.12). When (4.41) is applied to $\hat{Y}$ we obtain

$$
\hat{Y}=\tilde{r}_{1}(\hat{Y})+\tilde{r}_{2}(\hat{Y}) X+\tilde{W} \tilde{R}(\hat{Y}) .
$$


We now set $\tilde{Y}=\hat{Y}-r_{1}(\hat{Y})$ and obtain

$$
\tilde{Y}=c \tilde{X}+\tilde{W} g
$$

Since $\tilde{X} \tilde{Y}=\tilde{W}^{3}+\tilde{P}$ for some (modified) polynomial we can combine these two equations to obtain the desired relation between $\tilde{X}$ and $\tilde{W}$. The estimates on $g$ follow from the fact that $\tilde{g}=T f+O(\varepsilon)$ and estimates using the map $\tau$. The proof is finished the same way that that of Propositions 4.4 was. To follow that pattern, we need to know that the geometric configurations are the same. This follows from a local analytic geometric analysis of $\tilde{E}_{\theta}$. (We need to know that $\tilde{E}_{\theta}$ is connected to know that the map $L$ is onto some $\tilde{E}_{\theta}$.) This finishes the proof outline.

The geometric content of this proposition is the same as that of Proposition 4.4. One can see this by analyzing the equation defining $\tilde{E}_{\theta}$ or alternatively by regarding $\tilde{E}_{\theta}$ as a deformation (in the sense of analytic geometry) of the curve singularity given at the origin in $\mathbf{C}^{2}$ by $E_{\theta}$ and using the theory of deformation of curve singularities.

An explicit example of an algebra $B$ to which the previous Proposition applies is given in Proposition 2 of [14].

If $A$ is in $\mathscr{A}$ and the only singularities of $A$ are nodes and cusps then the results of this section can be combined to give a fairly complete description of algebras $B$ close to $A$. For example, if $B$ is close to $A$ then the only singularities of $B$ are nodes and cusps (and thus the set of algebras with only such singularities is open). Also, both the number of cusps on $B$ and total number of singular points of $B$ are dominated by the corresponding quantities for $A$.

Combining the results of this section with the counting formulas (2.1) and (2.2) and the constructions of [14] we can obtain a fairly complete description of elements in the neighborhoods of points of $\mathscr{A}_{k}$ for very small $k$.

First we introduce a bit more notation. For integers $r, j, k$ with $1 \leq r \leq j \leq k+1$ let

$$
\mathscr{A}_{k, j, r}=\left\{A \in \mathscr{A}_{k} ; \partial A \text { has } j\right. \text { components and }
$$

$$
M(A) \backslash \operatorname{Sing}(A) \text { has } r \text { components }\} .
$$

Let $\mathscr{A}_{k, j}=\cup_{r} \mathscr{A}_{k, j, r}$. We noted in Proposition 2.5 that $\mathscr{A}_{k, j}$ is empty if $k-j$ is even.

By [16], $\mathscr{A}_{0}$ is an isolated point of $\mathfrak{M}$.

$\mathscr{A}_{1}=\mathscr{A}_{1,2}=\mathscr{A}_{1,2,1} \cup \mathscr{A}_{1,2,2}$. First consider an $A$ in $\mathscr{A}_{1,2,1} \cdot M(A)$ is doubly connected and hence can be taken to be $S_{r}=\{z ; r \leq|z| \leq 1\}$ for some $r, 0<r<1$. By (2.1) we must have $A=A\left(S_{r}\right)$. By Theorem 4.1, $A$ has a neighborhood consisting of other $A\left(S_{t}\right)$ for $t$ near $r$. Now suppose $A$ 
is in $\mathscr{A}_{1,2,2}$. In this case $M(A)$ must consist of two simply connected Riemann surfaces with a pair of points identified. By composing with conformal automorphisms we may assume that the algebras are both copies of the disk algebra and that the identified points are the two origins. Thus $A$ is the subalgebra of functions in the direct sum of two copies of the disk algebra consisting of functions which take the same value at the two centers. By Proposition 4.4, any close neighbor of this $A$ is either of the same sort (and hence is isomorphic to this $A$ ) or is of the type $A\left(S_{r}\right)$ for small $r$. Proposition 1 of [14] shows that both possibilities occur. In particular we note that $\mathscr{A}_{1,2,1}$ is dense in $\mathscr{A}_{1}$.

We now describe $\mathscr{A}_{2} \cdot \mathscr{A}_{2}=\mathscr{A}_{2,1} \cup \mathscr{A}_{2,3}$. Since nearby algebras have homeomorphic Shilov boundaries, $d\left(\mathscr{A}_{2,1}, \mathscr{A}_{2,3}\right)>0$; hence we describe the two separately.

$\mathscr{A}_{2,1}$ contains three types of algebras. First there are algebras of the form $A(S)$ with $S$ in $\mathscr{S}, S$ a finite bordered Riemann surface of genus one with one boundary component (i.e. a torus with a hold cut out). By Theorem 4.1, any such $A$ has an open neighborhood consisting entirely of algebras of the same sort. The other possibilities for elements of $\mathscr{A}_{2,1}$ are subalgebras of codimension one of the disk algebra $A(D)$ (see (2.1)). If such an $A$ is obtained by a point identification then it is equivalent to an algebra $A_{r}=\{f \in A(D) ; f(0)=f(r)\}$ for some $r, 0<r<1$. By Proposition 4.7, a small neighborhood of $A_{r}$ contains algebras of the form $A(S)$ for $S$ as just described and algebras of the form $A_{t}$ for some $t, 0<t<1$. The analysis in Example 7.9 of [15] shows that $t$ would have to be close to $r$ and that example together with the constructions in [14] show that both possibilities occur. Finally we consider the algebra $A_{0}=\{f \in A(D)$; $\left.f^{\prime}(0)=0\right\}$. Proposition 4.6 allows the possibility that arbitrarily small neighborhoods of $A_{0}$ will contain algebras of the sort $A_{r}$ (for small $r$ ) and algebras of the sort $A(S)$ for $S$ in $\mathscr{S}$ as described. The example in [15] and constructions in [14] show that both possibilities occur in every neighborhood of $A_{0}$. Thus the algebras of the form $A(S), S$ in $\mathscr{S}$ form a dense subset of $\mathscr{A}_{2,1}$. Since it is known from Teichmüller theory that the moduli space for surfaces in $\mathscr{S}$ of fixed topological type is connected, $A(\mathscr{S}) \cap \mathscr{A}_{2,1}$ is connected. Thus $\mathscr{A}_{2,1}$ is connected. Also note that the set $\left\{A_{r} ; 0 \leq r \leq 1\right\}$ is a closed subset of $\mathscr{A}_{2,1}$.

We now consider $\mathscr{A}_{2,3} \cdot \mathscr{A}_{2,3}=\mathscr{A}_{2,3,1} \cup \mathscr{A}_{2,3,2} \cup \mathscr{A}_{2,3,3}$. If $A$ is in $\mathscr{A}_{2,3,1}$ then $A=A(S)$ for an $S$ in $\mathscr{S}$ of genus zero and with three boundary contours. Such an $S$ is conformally equivalent to a ring with a disk removed, i.e.,

$$
S=\{z ; r \leq|z| \leq 1\} \backslash\left\{z ;\left|z-r_{1}\right|<\delta\right\}
$$


where $r, r_{1}, \delta>0, r<r_{1}-\delta, r_{1}+\delta<1$. By Theorem 4.1, $A$ has a full neighborhood consisting of algebras of the same type. By Teichmüller theory the associated surfaces can be realized in the way just described and with nearby parameters $\tilde{r}, \tilde{r}_{1}, \tilde{\delta}$. If $A$ is in $\mathscr{A}_{2,3,2}$ then $A$ must be a subalgebra of codimension one of the sum $A\left(S_{r}\right) \oplus A(D)$ which is obtained by identifying a point of $S_{r}$ (which we may assume to be real and positive) and a point of $D$ (which we may take to be the origin). By Proposition 4.4 any such $A$ has a neighborhod consisting entirely of other such algebras and algebras in $\mathscr{A}_{2,3,1}$. Again, both possibilities occur in every neighborhood of such an $A$. Finally we consider the algebras $A$ in $\mathscr{A}_{2,3,3}$. By (2.1) such an $A$ must be obtained from a direct sum of three copies of the disk algebra by making two point identifications. One possibility is that the points which are identified were two distinct pairs of points. Such algebras could be realized, for instance, as algebras of continuous boundary value analytic functions on

$$
\{z ;|z| \leq 1\} \cup\{z ;|z-3| \leq 1\} \cup\{z ;|z-5| \leq 1\}
$$

which identify 0 and 3 and $3+r$ with 5 for some $r, 0<r<1$. This algebra has two nodes and no other singularites. Thus Theorem 4.2 and Proposition 4.4 apply and we find that all the algebras near such an $A$ are either of the same type or are elements of $\mathscr{A}_{2,3,2}$ or of $\mathscr{A}_{2,3,1}$. Both possibilities occur. Finally there is the possibility that the point identifications involved three points, not four. The only such $A$ is the subalgebra of a direct sum of three copies of the disk algebra obtained by identifying the three origins. (That is, the case $r=0$ in the construction just described.) This singularity is not a node or a cusp. (In fact, it is not a complete intersection.) Deformation of this type of singularity is discussed in Examples 4 and 9 of [9]. It is possible to prove an analog of Proposition 4.4 for this singularity. For now we just note that straightforward variations on the constructions on [14] and [15] show that every neighborhood of this algebra contains algebras of all the other types described for $\mathscr{A}_{2,3}$. Using the fact that $\operatorname{dim} H^{1}(M(A), R)$ is semi-continuous, (Corollary 7.7 of [15]) we see that $\mathscr{A}_{2,3,3}$ and $\mathscr{A}_{2,3,3} \cup \mathscr{A}_{2,3,2}$ are closed subset of $\mathscr{A}_{2,3}$. Since $\mathscr{A}_{2,3,1}$ is connected and dense, $\mathscr{A}_{2,3}$ is connected.

On the basis of this analysis, we speculate the $\mathscr{A}_{k, j}$ are always connected.

Conjecture 4.8. The connected components of $\mathscr{A}_{k}$ are exactly the non-trivial $\mathscr{A}_{k, j}$.

The analysis just given shows that the conjecture is true for $k=0,1,2$. The same type of analysis works for $k=3$. For $k=4, \mathscr{A}_{4}=\mathscr{A}_{4,5} \cup \mathscr{A}_{4,3}$ $\cup \mathscr{A}_{4,1}$. Analyses similar to the ones given work for $\mathscr{A}_{4,5}$ and $\mathscr{A}_{4,3}$. For $\mathscr{A}_{4,1}$, 
new techniques are needed (for example, to show that $\{f \in \mathscr{A}(D)$; $\left.f^{\prime}(0)=f^{\prime \prime}(0)=0\right\}$ and $\left\{f \in A(D) ; f^{\prime}(0)=f^{\prime \prime \prime}(0)=0\right\}$ are in the same component of $\mathscr{A}_{4,1}$ i.e., the component containing those $A$ with $\operatorname{Sing}(A)=$ $\varnothing$.) In this particular case, elementary ad hoc considerations suffice and the conjecture is true for $k=4$. We have not pursued the issue further.

By Theorem 4.1, those $A$ with $\operatorname{Sing}(A)=\varnothing$ form an open set. In the examples just described, this set is dense in the component which contains it. This suggests

Conjecture 4.9. For each $k,\left\{A \in \mathscr{A}_{k} ; \operatorname{Sing}(A)=\varnothing\right\}$ is dense in $\mathscr{A}_{k}$; i.e. $A(\mathscr{S}) \cap \mathscr{A}_{k}$ is dense in $\mathscr{A}_{k}$.

The analysis described for $k=0,1,2$ extends to $k=3$ and the conjecture is true for $0 \leq k \leq 3$. The intuitive content of the conjecture - that all elements of $\mathscr{A}$ are limits of elements of $A(\mathscr{S})$-is attractive. Furthermore the failure of the conjecture would leave open the problem of describing $\overline{A(\mathscr{S})}$. However, an analogous conjecture in algebraic deformation theory is known to be false [8]. (Since our theory is based on small deformations rather than infinitesimal deformations, it is not clear how much evidence is provided by that failure.)

It should be pointed out that the description given of $\mathscr{A}_{2}$ was in terms of the topology of $M(B)$ for $B$ near $A$. We have still not given a description of the conformal geometry. If $\operatorname{Sing}(A)$ is empty, then Theorem 4.1 is decisive (even if not explicit). Consider, however, for example, the algebra $A=\{f \in A(D) ; f(0)=f(1 / 2)\}$. We noted that neighborhoods of this algebra contain algebras $A_{t}=\{f \in A(D) ; f(0)=f(t)\}$ for some $t$, $0<t<1$. The fact that $t$ will be near $1 / 2$ for small neighborhoods of $A$ uses the continuity of the successive minima of the normed integer cohomology group (Theorem 7.2 of [15]). We also know that neighborhoods of this same $A$ contain algebras of the form $A(S)$ for $S$ in $\mathscr{S}, S$ of genus one with one boundary contour. We do not have a convenient description of which $S$ arise this way. (The continuity of successive minima gives some information, but not an easy to understand description.)

\section{REFERENCES}

[1] L. V. Ahlfors, Open Riemann surfaces and extremal problems on compact subregions, Comment. Math. Helv., 24 (1950), 100-134.

[2] T. W. Gamelin, Uniform Algebras, Prentice-Hall, Englewood Cliffs, N. J. 1969.

[3] _ Embedding Riemann surfaces in maximal ideal spaces, J. Funct. Anal., 2 (1968), 123-146. 
[4] M. Gerstenhaber, On the deformation of rings and algebras, Ann. of Math., 79 (1964), 59-103.

[5] B. E. Johnson, Cohomology in Banach algebras, Memoirs, Amer. Math. Soc., 127 (Providence, R. I. 1972).

[6] _ Perturbation of Banach algebras, Proc. London Math. Soc., (3) 35 (1977), 439-458.

[7] O. Lehto and K. I. Virtanen, Quasiconformal Mappings in the Plane, 2nd ed., Springer-Verlag, Berlin, 1973.

[8] D. Mumford, Pathologies IV, Amer. J. Math., 97 (1975), 847-849.

[9] V. P. Palamodov, Deformation of complex spaces, Russian Math. Surveys, 31.3 (1976), 129-197.

[10] I. Raeburn and J. L. Taylor, Hochschild cohomology and perturbation of Banach algebras, J. Functional Anal., 25 (1977), 258-267.

[11] R. Rochberg, Almost isometries of Banach spaces and moduli of Riemann surfaces, Duke Math. J., 40 (1973), 41-52.

[12] __ Almost Isometries of Banach Spaces and moduli of Riemann surfaces II, Duke Math. J., 42 (1975), 167-182.

[13] Algebras of analytic functions on degenerating Riemann surfaces, Bull. Amer. Math. Soc., 81 (1975), 202-204.

[14] _ The Banach-Mazur distance between function algebras on degenerating Riemann surfaces, Lecture Notes in Mathematics 604, (1977), 82-94.

[15] _ Deformation of uniform algebras, Proc. London Math. Soc., (3) 39 (1979), 93-118.

[16] The disk algebra is rigid, Proc. London Math. Soc., (3) 39 (1979), 119-130.

[17] E. L. Stout, The Theory of Uniform Algebras, Bogden and Quigley, Belmont, California, 1971.

[18] J. Wermer, Banach Algebras and Several Complex Variables, 2nd ed., Springer-Verlag, New York, 1976.

[19] K. Jarosz, Perturbation of Banach Algebras, Lecture Notes in Mathematics, 1120 (1985).

Received January 30, 1985. This work supported by NSF Grant MCS 7903122.

WASHINGTON UNIVERSITY

ST. LouIS, MO 63130 



\section{PACIFIC JOURNAL OF MATHEMATICS EDITORS}

V. S. VARADARAJAN (Managing Editor)
University of California
Los Angeles, CA 90024
HEBERT CLEMENS
University of Utah
Salt Lake City, UT 84112
CHARLES R. DEPRIMA
California Institute of Technology
Pasadena, CA 91125

\section{R. FINN}

Stanford University

Stanford, CA 94305

HeRmanN FlaschKa

University of Arizona

Tucson, AZ 85721

RAMESH A. GANGOLli

University of Washington

Seattle, WA 98195

ROBION KIRBY

University of California

Berkeley, CA 94720

\section{C. MoOre}

University of California

Berkeley, CA 94720

H. SAMELSON

Stanford University

Stanford, CA 94305

HAROLD STARK

University of California, San Diego

La Jolla, CA 92093

ASSOCIATE EDITORS
R. ARENS
E. F. BECKENBACH
B. H. NeUmanN
F. WOLF
K. Yoshida (1906-1982)

\section{SUPPORTING INSTITUTIONS}

UNIVERSITY OF ARIZONA

UNIVERSITY OF BRITISH COLUMBIA

CALIFORNIA INSTITUTE OF TECHNOLOGY

UNIVERSITY OF CALIFORNIA

MONTANA STATE UNIVERSITY

UNIVERSITY OF NEVADA, RENO

NEW MEXICO STATE UNIVERSITY

OREGON STATE UNIVERSITY
UNIVERSITY OF OREGON

UNIVERSITY OF SOUTHERN CALIFORNIA

STANFORD UNIVERSITY

UNIVERSITY OF HAWAII

UNIVERSITY OF TOKYO

UNIVERSITY OF UTAH

WASHINGTON STATE UNIVERSITY

UNIVERSITY OF WASHINGTON 


\section{Pacific Journal of Mathematics}

\section{Vol. 121, No. $1 \quad$ November, 1986}

Om P. Agrawal, Douglas Napier Clark and Ronald George Douglas,

Invariant subspaces in the polydisk $\ldots \ldots \ldots \ldots \ldots \ldots \ldots \ldots \ldots \ldots$

Christoph Bandt and Gebreselassie Baraki, Metrically invariant measures

on locally homogeneous spaces and hyperspaces $\ldots \ldots \ldots \ldots \ldots \ldots \ldots$

Marcy Mason Barge, Horseshoe maps and inverse limits ..............29

Russell Gene Bilyeu, Robert Richard Kallman and Paul Weldon Lewis,

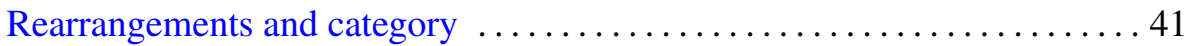

Jean Bourgain, A problem of Douglas and Rudin on factorization . . .......47

Hernan Cendra, A normal form and integration in finite terms for a class of

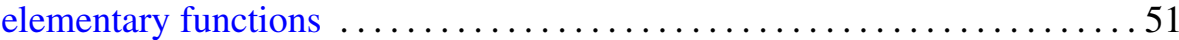

Ky Fan, The angular derivative of an operator-valued analytic function . . . . 67

Gerhard Gierz, On the Dunford-Pettis property of function modules of

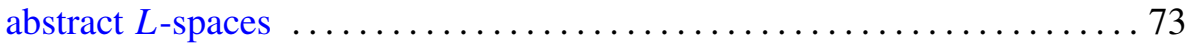

Gabriel Katz, On polynomial generators in the algebra of complex functions

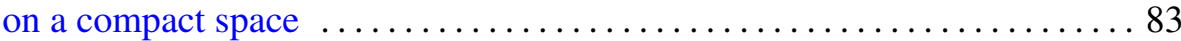

Ridgley Lange, Duality and asymptotic spectral decompositions $\ldots \ldots \ldots . .93$

Anthony To-Ming Lau and Peter F. Mah, Quasinormal structures for certain spaces of operators on a Hilbert space ................... 109

R. Daniel Mauldin, Correction: "The set of continuous nowhere differentiable functions"

Alan Harvey Mekler and Saharon Shelah, $\omega$-elongations and Crawley's problem

Alan Harvey Mekler and Saharon Shelah, The solution to Crawley's problem

Richard Rochberg, Deformation of uniform algebras on Riemann surfaces

Joseph Roitberg, On weak epimorphisms in homotopy theory

Jesús M. Ruiz, A remark on fields with the dense orbits property

Henry Wente, Counterexample to a conjecture of H. Hopf

David G. Wright, Rigid sets in $E^{n}$ 